\title{
ON POINTED HOPF ALGEBRAS WITH CLASSICAL WEYL GROUPS
}

\author{
SHOUCHUAN ZHANG
}

\begin{abstract}
Many cases of infinite dimensional Nichols algebras of irreducible YetterDrinfeld modules over classical Weyl groups are found. It is proved that except a few cases Nichols algebras of reducible Yetter-Drinfeld modules over classical Weyl groups are infinite dimensional. Some finite dimensional Nichols algebras of Yetter-Drinfeld modules over classical Weyl groups are given.
\end{abstract}

\section{INTRODUCTION}

This article is to contribute to the classification of finite-dimensional complex pointed Hopf algebras with Weyl groups of classical type. Weyl groups are very important in the theories of Lie groups, Lie algebras and algebraic groups. The classification of finite dimensional pointed Hopf algebra with finite abelian groups has been finished (see $\underline{\mathrm{He} 06}$, AS00]). Many cases of infinite dimensional Nichols algebras of irreducible Yetter-Drinfeld (YD) modules over symmetric group were discarded in [AFZ]. All -1-type pointed Hopf algebras with Weyl groups of exceptional type were found in [ZZWC] and it was showed that every non -1-type pointed Hopf algebra is infinite dimensional in [AZ07, ZZWC]. It was obtained that every Nichols algebra of reducible YD module over simple group and symmetric group is infinite dimensional in [HS]. Hopf subalgebras of co-path Hopf algebras was studied in OZ04.

In this paper we discard many cases of infinite dimensional Nichols algebras of irreducible YD modules over classical Weyl groups by mean of co-path Hopf algebras and the results of [AFZ]. [HS] said that if Nichols algebra of reducible YD module is finite dimensional, then their conjugacy classes are square-commutative (see [HS, Theorem 8.6]). We obtain that except a few cases Nichols algebras of reducible YD modules over classical Weyl groups are infinite dimensional by applying result of [HS]. We also find some finite dimensional Nichols algebras of YD modules over classical Weyl groups.

The main results in this paper are summarized in the following statements.

Theorem 1. Let $G=A \rtimes \mathbb{S}_{n}$ be a classical Weyl group with $A \subseteq\left(C_{2}\right)^{n}$ and $n>2$. Assume that $M=M\left(\mathcal{O}_{\sigma_{1}}, \rho^{(1)}\right) \oplus M\left(\mathcal{O}_{\sigma_{2}}, \rho^{(2)}\right) \oplus \cdots \oplus M\left(\mathcal{O}_{\sigma_{m}}, \rho^{(m)}\right)$ is a reducible YD module over $k G$.

(i) Assume that there exist $i \neq j$ such that $\sigma_{i}, \sigma_{j} \notin A$. If $\operatorname{dim} \mathfrak{B}(M)<\infty$, then $n=4$, the type of $\sigma_{p}$ is $2^{2}$ and the sign of $\sigma_{p}$ is stable for any $1 \leq p \leq m$ with $\sigma_{p} \notin A$. 
(ii) If $\sigma_{i}=a:=\left(g_{2}, g_{2}, \cdots, g_{2}\right) \in G$ and $\rho^{(i)}=\theta_{\chi^{\left(\nu_{i}\right)}, \mu^{(i)}}:=\left(\chi^{\left(\nu_{i}\right)} \otimes \mu^{(i)}\right) \uparrow_{G_{\chi\left(\nu_{i}\right)}^{a}}^{G^{a}} \in \widehat{G^{a}}$ with odd $\nu_{i}$ for $i=1,2, \cdots, m$, then $\mathfrak{B}(M)$ is finite dimensional.

Theorem 2. Let $0 \leq \nu \leq n$ and Let $G=A \rtimes \mathbb{S}_{n}$. Let $\sigma \in \mathbb{S}_{n}$ be of type $\left(1^{\lambda_{1}}, 2^{\lambda_{2}}, \ldots, n^{\lambda_{n}}\right)$ and $\rho=\rho^{\prime} \otimes \rho^{\prime \prime} \in \widehat{\left(\widehat{\left.\mathbb{S}_{n}\right)_{\chi}^{\sigma}(\nu)}\right.}$ with $\rho^{\prime} \in \widehat{\mathbb{S}_{\nu}^{\sigma}}$ and $\rho^{\prime \prime} \in{\mathbb{\mathbb { S } ^ { \sigma }}}_{\{\nu+1, \cdots, n\}}$. Assume that $\mathfrak{B}\left(\mathcal{O}_{\sigma}^{G}, \theta_{\chi^{(\nu)}, \rho}\right)$ is matched with $\operatorname{dim} \mathfrak{B}\left(\mathcal{O}_{\sigma}^{G}, \theta_{\chi^{(\nu)}, \rho}\right)<\infty$. Let $\mu=\otimes_{1 \leq i \leq n} \mu_{i}$ with $\mu_{i}:=\theta_{\chi^{\mathbf{t}_{i}, \rho_{i}}}$ as in (2.2) denote $\rho^{\prime}$ when $\sigma \in \mathbb{S}_{\nu}$ and $\rho^{\prime \prime}$ when $\sigma \in \mathbb{S}_{\{\nu+1, \nu+2, \cdots, n\}}$, respectively. Let $\lambda_{1}^{\prime}=\lambda_{1}-(n-\nu)$ when $\sigma \in \mathbb{S}_{\nu} ; \lambda_{1}^{\prime}=\lambda_{1}-\nu$ when $\sigma \in \mathbb{S}_{\{\nu+1, \nu+2, \cdots, n\}}$. Then some of the following hold:

(i) $\left(1^{\lambda_{1}^{\prime}}, 2\right), \mu_{1}=\operatorname{sgn}$ or $\epsilon, \mu_{2}=\chi_{(1 ; 2)}$.

(ii) $\left(2, \sigma_{o}\right), \sigma_{o}:=\prod_{1 \leq i \leq n, 1<i \text { is odd }} \sigma_{i} \neq \mathrm{id}, \mu_{2}=\chi_{(1 ; 2)}, \mu_{j}=\left(\chi_{(0, \ldots, 0 ; j)} \otimes \rho_{j}\right) \uparrow_{\left(\mathbb{S}_{Y_{j}}\right)_{\chi_{(0, \ldots, 0 ; j)}}^{\sigma_{j}}}^{\left(\mathbb{S}_{Y_{j}} \sigma_{j}^{\sigma_{j}}\right.}$, for all odd $j>1$.

(iii) $\left(1^{\lambda_{1}^{\prime}}, 2^{3}\right), \mu_{1}=\operatorname{sgn}$ or $\epsilon, \mu_{2}=\chi_{(1,1,1 ; 2)} \otimes \epsilon$ or $\chi_{(1,1,1 ; 2)} \otimes \operatorname{sgn}$.

Furthermore, if $\lambda_{1}^{\prime}>0$, then $\mu_{2}=\chi_{(1,1,1 ; 2)} \otimes \operatorname{sgn}$.

(iv) $\left(2^{5}\right), \mu_{2}=\chi_{(1,1,1,1,1 ; 2)} \otimes \epsilon$ or $\chi_{(1,1,1,1,1 ; 2)} \otimes \operatorname{sgn}$.

(v) $\left(1^{\lambda_{1}^{\prime}}, 4\right), \mu_{1}=\operatorname{sgn}$ or $\epsilon, \mu_{4}=\chi_{(2 ; 4)}$.

(vi) $\left(1^{\lambda_{1}^{\prime}}, 4^{2}\right), \mu_{1}=\operatorname{sgn}$ or $\epsilon, \mu_{4}=\chi_{(1,1 ; 4)} \otimes \operatorname{sgn}$ or $\chi_{(3,3 ; 4)} \otimes \operatorname{sgn}$.

(vii) $(2,4), \mu_{2}=\chi_{(1 ; 2)}$ and $\mu_{4}=\epsilon$ or $\mu_{2}=\epsilon$ and $\mu_{4}=\chi_{(2 ; 4)}$.

(viii) $\left(2,4^{2}\right), \mu_{2}=\epsilon, \mu_{4}=\chi_{(1,1 ; 4)} \otimes \operatorname{sgn}$ or $\chi_{(3,3 ; 4)} \otimes \operatorname{sgn}$.

(ix) $\left(2^{2}, 4\right), \operatorname{deg} \mu_{2}=1, \mu_{4}=\chi_{(2 ; 4)}$.

Furthermore, $\mathfrak{B}\left(\mathcal{O}_{\sigma}^{G}, \theta_{\chi^{(\nu)}, \rho}\right)$ is -1-type under the cases above.

Indeed, Theorem 1 follows Remark 3.8 and Remark 3.12. The proof of Theorem 2 is in subsection 3.1 .

\section{Preliminaries And Conventions}

Let $k$ be the complex field and $G$ a finite group. Let $\hat{G}$ denote the set of all isomorphic classes of irreducible representations of group $G, G^{\sigma}$ the centralizer of $\sigma, \mathcal{O}_{\sigma}$ or $\mathcal{O}_{\sigma}^{G}$ the conjugacy class in $G, C_{j}$ the cycle group with order $j, g_{j}$ a generator of $C_{j}$ and $\chi_{j}$ a character of $C_{j}$ with order $j$. The Weyl groups of $A_{n}, B_{n}, C_{n}$ and $D_{n}$ are called the classical Weyl groups. Let $\rho \uparrow G$ denote the induced representation of $\rho$ as in [Sa01].

A quiver $Q=\left(Q_{0}, Q_{1}, s, t\right)$ is an oriented graph, where $Q_{0}$ and $Q_{1}$ are the sets of vertices and arrows, respectively; $\sigma$ and $t$ are two maps from $Q_{1}$ to $Q_{0}$. For any arrow $a \in Q_{1}$, $s(a)$ and $t(a)$ are called its start vertex and end vertex, respectively, and $a$ is called an arrow from $s(a)$ to $t(a)$. For any $n \geq 0$, an $n$-path or a path of length $n$ in the quiver $Q$ is an ordered sequence of arrows $p=a_{n} a_{n-1} \cdots a_{1}$ with $t\left(a_{i}\right)=s\left(a_{i+1}\right)$ for all $1 \leq i \leq n-1$. Note that a 0 -path is exactly a vertex and a 1 -path is exactly an arrow. In this case, we define $s(p)=s\left(a_{1}\right)$, the start vertex of $p$, and $t(p)=t\left(a_{n}\right)$, the end vertex of $p$. For a 0 -path $x$, we have $s(x)=t(x)=x$. Let $Q_{n}$ be the set of $n$-paths. Let ${ }^{y} Q_{n}^{x}$ denote the set of all $n$-paths from $x$ to $y, x, y \in Q_{0}$. That is, ${ }^{y} Q_{n}^{x}=\left\{p \in Q_{n} \mid s(p)=x, t(p)=y\right\}$. 
A quiver $Q$ is finite if $Q_{0}$ and $Q_{1}$ are finite sets. A quiver $Q$ is locally finite if ${ }^{y} Q_{1}^{x}$ is a finite set for any $x, y \in Q_{0}$.

Let $G$ be a group. Let $\mathcal{K}(G)$ denote the set of conjugate classes in $G$. A formal sum $r=\sum_{C \in \mathcal{K}(G)} r_{C} C$ of conjugate classes of $G$ with cardinal number coefficients is called a ramification (or ramification data) of $G$, i.e. for any $C \in \mathcal{K}(G), r_{C}$ is a cardinal number. In particular, a formal sum $r=\sum_{C \in \mathcal{K}(G)} r_{C} C$ of conjugate classes of $G$ with non-negative integer coefficients is a ramification of $G$.

For any ramification $r$ and a $C \in \mathcal{K}(G)$, since $r_{C}$ is a cardinal number, we can choice a set $I_{C}(r)$ such that its cardinal number is $r_{C}$ without loss of generality. Let $\mathcal{K}_{r}(G):=$ $\left\{C \in \mathcal{K}(G) \mid r_{C} \neq 0\right\}=\left\{C \in \mathcal{K}(G) \mid I_{C}(r) \neq \emptyset\right\}$. If there exists a ramification $r$ of $G$ such that the cardinal number of ${ }^{y} Q_{1}^{x}$ is equal to $r_{C}$ for any $x, y \in G$ with $x^{-1} y \in C \in \mathcal{K}(G)$, then $Q$ is called a Hopf quiver with respect to the ramification data $r$. In this case, there is a bijection from $I_{C}(r)$ to ${ }^{y} Q_{1}^{x}$, and hence we write ${ }^{y} Q_{1}^{x}=\left\{a_{y, x}^{(i)} \mid i \in I_{C}(r)\right\}$ for any $x, y \in G$ with $x^{-1} y \in C \in \mathcal{K}(G)$.

$\operatorname{deg} \rho$ denotes the dimension of the representation space $V$ for a representation $(V, \rho)$.

Recall the notation RSR in [ZCZ, Def. 1.1]. Let $\rho_{C}$ be a representation of $G^{u(C)}$ with irreducible decomposition $\rho=\oplus_{i \in I_{C}(r, u)} \rho_{C}^{(i)}$, where $I_{C}(r, u)$ is an index set. Let $\vec{\rho}$ denote $\left\{\rho_{C}\right\}_{C \in \mathcal{K}_{r}(G)}=\left\{\left\{\rho_{C}^{(i)}\right\}_{i \in I_{C}(r, u)}\right\}_{C \in \mathcal{K}_{r}(G)} . \quad(G, r, \vec{\rho}, u)$ is called an RSR when $\operatorname{deg}\left(\rho_{C}\right)=r_{C}$ for any $C \in \mathcal{K}_{r}(G)$, written as $\operatorname{RSR}(G, r, \vec{\rho}, u)$. For any $\operatorname{RSR}(G, r, \vec{\rho}, u)$, we obtain a co-path Hopf algebra $k Q^{c}(G, r, \vec{\rho}, u)$, a Hopf algebra $k G\left[k Q_{1}^{c}, G, r, \vec{\rho}, u\right]$ of one type, a $k G$-YD module $\left(k Q_{1}^{1}, a d(G, r, \vec{\rho}, u)\right)$ and a Nicolas algebra $\mathfrak{B}(G, r, \vec{\rho}, u):=$ $\mathfrak{B}\left(k Q_{1}^{1}, a d(G, r, \vec{\rho}, u)\right)$ (see [ZCZ]).

If ramification $r=r_{C} C$ and $\left|I_{C}(r, u)\right|=1$ then we say that $\operatorname{RSR}(G, r, \vec{\rho}, u)$ is of bi-one since $r$ only has one conjugacy class $C$ and $\left|I_{C}(r, u)\right|=1$. Furthermore, if let $\sigma=u(C)$, $C=\mathcal{O}_{\sigma}, r_{C}=m$ and $\rho_{C}^{(i)}=\rho$ for $i \in I_{C}(r, u)$, then bi-one $\operatorname{RSR}(G, r, \vec{\rho}, u)$ is denoted by $\operatorname{RSR}\left(G, m \mathcal{O}_{\sigma}, \rho\right)\left(\right.$ or $\left.\operatorname{RSR}\left(G, \mathcal{O}_{\sigma}, \rho\right)\right)$, in short.

$\operatorname{RSR}(G, r, \vec{\rho}, u)$ is called to be -1 -type, if $u(C)$ is real (i.e. $\left.u(C)^{-1} \in C\right)$ and the order of $u(C)$ is even with $\rho_{C}^{(i)}(u(C))=-$ id for any $C \in \mathcal{K}_{r}(G)$ and any $i \in I_{C}(r, u)$. In this case, the Nichols algebra $\mathfrak{B}(G, r, \vec{\rho}, u)$ is called to be -1-type.

For $s \in G$ and $(\rho, V) \in \widehat{G^{s}}$, here is a precise description of the YD module $M\left(\mathcal{O}_{s}, \rho\right)$, introduced in Gr00, AZ07]. Let $t_{1}=s, \ldots, t_{m}$ be a numeration of $\mathcal{O}_{s}$, which is a conjugacy class containing $s$, and let $g_{i} \in G$ such that $g_{i} \triangleright s:=g_{i} s g_{i}^{-1}=t_{i}$ for all $1 \leq i \leq m$. Then $M\left(\mathcal{O}_{s}, \rho\right)=\oplus_{1 \leq i \leq m} g_{i} \otimes V$. Let $g_{i} v:=g_{i} \otimes v \in M\left(\mathcal{O}_{s}, \rho\right), 1 \leq i \leq m$, $v \in V$. If $v \in V$ and $1 \leq i \leq m$, then the action of $h \in G$ and the coaction are given by

$$
\delta\left(g_{i} v\right)=t_{i} \otimes g_{i} v, \quad h \cdot\left(g_{i} v\right)=g_{j}(\gamma \cdot v),
$$

where $h g_{i}=g_{j} \gamma$, for some $1 \leq j \leq m$ and $\gamma \in G^{s}$. Let $\mathfrak{B}\left(\mathcal{O}_{s}, \rho\right)$ denote $\mathfrak{B}\left(M\left(\mathcal{O}_{s}, \rho\right)\right)$. By [ZZWC, Lemma 1.1], there exists a bi-one arrow Nichols algebra $\mathfrak{B}(G, r, \vec{\rho}, u)$ such that $\mathfrak{B}\left(\mathcal{O}_{s}, \rho\right) \cong \mathfrak{B}(G, r, \vec{\rho}, u)$ as graded braided Hopf algebras in ${ }_{k G}^{k G} \mathcal{Y} D$. 
If $D$ is a subgroup of $G$ and $C$ is a congugacy class of $D$, then $C_{G}$ denotes the conjugacy class of $G$ containing $C$.

\section{1. $G=A \rtimes D$}

In this section we give the relation between Nichols algebras over group $A \rtimes D$ and group $D$.

Let $G=A \rtimes D$ be a semidirect product of abelian group $A$ and group $D$. For any $\chi \in \hat{A}$, let $D_{\chi}:=\{h \in D \mid h \cdot \chi=\chi\} ; G_{\chi}:=A \rtimes D_{\chi}$. For an irreducible representation $\rho$ of $D_{\chi}$, let $\theta_{\chi, \rho}:=(\chi \otimes \rho) \uparrow_{G_{\chi}}^{G}$, the induced representation of $\chi \otimes \rho$ on $G$. By [Se, Pro.25], every irreducible representation of $G$ is of the following form: $\theta_{\chi, \rho}$. Let $\epsilon \in \hat{A}$ with $\epsilon(a)=1$ for any $a \in A$. Thus $D_{\epsilon}=D$ and $\theta_{\epsilon, \rho}$ is an irreducible representation of $G$.

Lemma 1.1. Let $G=A \rtimes D$ and $\sigma \in D$. Then $G^{\sigma}=A^{\sigma} \rtimes D^{\sigma}$.

Proof. If $x=(a, d) \in G^{\sigma}$, then $x \sigma=\sigma x$. Thus

$$
a=\sigma \cdot a \text { and } \quad d \sigma=\sigma d
$$

This implies $d \in D^{\sigma}$ and $a \in A^{\sigma}$ since $\sigma \cdot a=\sigma a \sigma^{-1}$.

Conversely, if $x=(a, d) \in A^{\sigma} \rtimes D^{\sigma}$, then (1.1) holds. This implies $x \sigma=\sigma x$ and $x \in G^{\sigma}$.

Lemma 1.2. Let $D$ be a subgroup of $G$ with $\sigma \in D$ and let right coset decompositions of $D^{\sigma}$ in $D$ be

$$
D=\bigcup_{\theta \in \Theta} D^{\sigma} g_{\theta}
$$

Then there exists a set $\Theta^{\prime}$ with $\Theta \subseteq \Theta^{\prime}$ such that

$$
G=\bigcup_{\theta \in \Theta^{\prime}} G^{\sigma} g_{\theta}
$$

is a right coset decompositions of $G^{\sigma}$ in $G$.

Proof. For any $h, g \in D$, It is clear that $h g^{-1} \in D^{\sigma}$ if and only if $h g^{-1} \in G^{\sigma}$, which prove the claim.

Lemma 1.3. If $k Q^{c}(G, r, \vec{\rho}, u)$ is a co-path Hopf algebra (see [ZZC, $\left.\mathrm{ZCZ}\right]$ ), then $k G+$ $k Q_{1}=\left(k G\left[k Q_{1}^{c}\right]\right)_{1}$, where $k G\left[k Q_{1}^{c}\right]:=k G\left[k Q_{1}^{c}, G, r, \vec{\rho}, u\right]$ and $\left(k G\left[k Q_{1}^{c}\right]\right)_{1}$ denotes the second term of the coradical filtration of $k G\left[k Q_{1}^{c}\right]$.

Proof. By [ZCZ, Lemma 2.2], $R:=\operatorname{diag}\left(k G\left[k Q_{1}^{c}\right]\right)$ is a Nichols algebra. AS98, Lemma $2.5]$ yields that $k G\left[k Q_{1}^{c}\right]$ is coradically graded. 
Definition 1.4. Let $D$ be a subgroup of $G ; r$ and $r^{\prime}$ ramifications of $D$ and $G$, respectively. If $r_{C} \leq r_{C_{G}}^{\prime}$ for any $C \in \mathcal{K}_{r}(D)$, then $r$ is called a subramification of $r^{\prime}$, written as $r \leq r^{\prime}$. Furthermore, if $u(C)=u^{\prime}\left(C_{G}\right), I_{C}(r, u) \subseteq I_{C_{G}}\left(r^{\prime}, u^{\prime}\right)$ and $\rho_{C}^{(i)}$ is isomorphic to a subrepresentation of the restriction of $\rho_{C_{G}^{\prime}}^{\prime(i)}$ on $D^{u(C)}$ for any $C \in \mathcal{K}_{r}(D), i \in I_{C}(r, u)$, then $\operatorname{RSR}(D, r, \vec{\rho}, u)$ is called a sub-RSR of $\operatorname{RSR}\left(G, r^{\prime}, \overrightarrow{\rho^{\prime}}, u^{\prime}\right)$, written as $\operatorname{RSR}(D, r, \vec{\rho}, u) \leq$ $\operatorname{RSR}\left(G, r^{\prime}, \overrightarrow{\rho^{\prime}}, u^{\prime}\right)$.

Lemma 1.5. Let $D$ be a subgroup of $G$. If $\sigma \in D$, then $\operatorname{RSR}\left(D, \mathcal{O}_{\sigma}, \rho\right) \leq \operatorname{RSR}\left(G, \mathcal{O}_{\sigma}, \rho^{\prime}\right)$ if and only if $\rho$ is isomorphic to subrepresentation of the restriction of $\rho^{\prime}$ on $D^{\sigma}$.

Proof. It follows from Definition 1.4.

Proposition 1.6. Let $D$ be a subgroup of $G$. If $\operatorname{RSR}(D, r, \vec{\rho}, u) \leq \operatorname{RSR}\left(G, r^{\prime}, \overrightarrow{\rho^{\prime}}, u^{\prime}\right)$, then

(i) $k Q^{c}(D, r, \vec{\rho}, u)$ is a Hopf subalgebra $k Q^{\prime c}\left(G, r^{\prime}, \overrightarrow{\rho^{\prime}}, u^{\prime}\right)$.

(ii) $k D\left[k Q^{c}, D, r, \vec{\rho}, u\right]$ is a Hopf subalgebra $k G\left[k Q^{\prime c}, G, r^{\prime}, \overrightarrow{\rho^{\prime}}, u^{\prime}\right]$.

(iii) If $\mathfrak{B}\left(G, r^{\prime}, \overrightarrow{\rho^{\prime}}, u^{\prime}\right)$ is finite dimensional with finite group $G$ then so is $\mathfrak{B}(D, r, \vec{\rho}, u)$.

Proof. (i) For any $C \in \mathcal{K}_{r}(D)$ and $i \in I_{C}(r, u)$, let $X_{C}^{(i)}$ be a representation space of $\rho_{C}^{(i)}$ with a basis $\left\{x_{C}^{(i, j)} \mid j \in J_{C}(i)\right\}$ and $X_{C_{G}^{\prime}}^{(i)}$ a representation space of $\rho_{C_{G}}^{(i)}$ with a basis $\left\{x^{\prime(i, j)} \mid j \in J_{C_{G}}(i)\right\}$ and $J_{C}(i) \subseteq J_{C_{G}}(i) . \psi_{C}^{(i)}$ is a $k D^{u(C)}$-module monomorphism from $X_{C}^{(i)}$ to $X_{C_{G}}^{\prime(i)}$ with $x_{C_{G}}^{(i, j)}=\psi_{C}^{(i)}\left(x_{C}^{(i, j)}\right)$ for $i \in I_{C}(r, u), j \in J_{C}(i)$.

Let $\phi$ be an inclusion map from $k D$ to $k G$ and $\psi$ is a map from $k Q_{1}^{c}(D, r, \vec{\rho}, u)$ to $k Q_{1}^{\prime c}\left(G, r^{\prime}, \overrightarrow{\rho^{\prime}}, u^{\prime}\right)$ by sending $a_{y, x}^{(i, j)}$ to $a_{y, x}^{(i, j)}$ for any $y, x \in D, i \in I_{C}(r, u), j \in J_{C}(i)$ with $x^{-1} y \in C \in \mathcal{K}_{r}(D)$. Now we show that $\psi$ is a $k D$-bimodule homomorphism from $k Q_{1}^{c}$ to ${ }_{\phi}\left(k Q_{1}^{\prime c}\right)_{\phi}$ and a $k G$-bicomodule homomorphism from ${ }^{\phi}\left(k Q_{1}^{c}\right)^{\phi}$ to $k Q_{1}^{\prime c}$. We only show this about right modules since the others are similar. For any $h \in D, C \in \mathcal{K}_{r}(D)$, $i \in I_{C}(r, u), j \in J_{C}(i), x, y \in D$ with $x^{-1} y=g_{\theta}^{-1} u(C) g_{\theta}$ and $g_{\theta} h=\zeta_{\theta}(h) g_{\theta^{\prime}}, \zeta_{\theta}(h) \in D^{\sigma}$, $\theta, \theta^{\prime} \in \Theta_{C} \subseteq \Theta_{C_{G}}$ (see Lemma 1.2), see

$$
\begin{aligned}
\psi\left(a_{y, x}^{(i, j)} \cdot h\right) & =\psi\left(\sum_{s \in J_{C}(i)} k_{C, h}^{(i, j, s)} a_{y h, x h}^{(i, s)}\right) \quad \text { by [ZCZ, Pro.1.2]) } \\
& =\sum_{s \in J_{C}(i)} k_{C, h}^{(i, j, s)} a_{y h, x h}^{\prime(i, s)} \text { and } \\
\psi\left(a_{y, x}^{(i, j)}\right) \cdot h & =a_{y, x}^{\prime(i, j)} \cdot h \\
& =\sum_{s \in J_{C_{G}}(i)} k_{C_{G}, h}^{(i, j, s)} a_{y h, x h}^{\prime(i, s)} \text { (by [ZCZ, Pro.1.2]), }
\end{aligned}
$$

where $x_{C}^{(i, j)} \cdot \zeta_{\theta}(h)=\sum_{s \in J_{C}(i)} k_{C, h}^{(i, j, s)} x_{C}^{(i, s)}, x_{C_{G}^{\prime}}^{(i, j)} \cdot \zeta_{\theta}(h)=\sum_{s \in J_{C_{G}}(i)} k_{C_{G}, h}^{(i, j, s)} x_{C_{G}^{\prime}}^{(i, s)}$. Since

$$
\begin{aligned}
x_{C_{G}}^{\prime(i, j)} \cdot \zeta_{\theta}(h) & =\psi_{C}^{(i)}\left(x_{C}^{(i, j)}\right) \cdot \zeta_{\theta}(h)=\psi_{C}^{(i)}\left(x_{C}^{(i, j)} \cdot \zeta_{\theta}(h)\right) \\
& =\psi_{C}^{(i)}\left(\sum_{s \in J_{C}(i)} k_{C, h}^{(i, j, s)} x_{C}^{(i, s)}\right)=\sum_{s \in J_{C}(i)} k_{C, h}^{(i, j, s)} x_{C_{G}^{\prime}}^{(i, s)},
\end{aligned}
$$


which implies $\psi\left(a_{y, x}^{(i, j)} \cdot h\right)=\psi\left(a_{y, x}^{(i, j)}\right) \cdot h$.

By [ZZC, Lemma 1.5], $T_{k G}^{c}\left(\phi \pi_{0}, \psi \pi_{1}\right):=\phi \pi_{0}+\sum_{n>0} T_{n}^{c}\left(\psi \pi_{1}\right) \Delta_{n-1}$ is a graded Hopf algebra map from $T_{k D}^{c}\left(k Q_{1}^{c}\right)$ to $T_{k G}^{c}\left(k Q_{1}^{\prime c}\right)$. By Lemma 1.3, $\left(T_{k D}^{c}\left(k Q_{1}^{c}\right)\right)_{1}=k D+k Q_{1}^{c}$. Since the restriction of $T_{k G}^{c}\left(\phi \pi_{0}, \psi \pi_{1}\right)$ on $\left(T_{k D}^{c}\left(k Q_{1}^{c}\right)\right)_{1}$ is $\phi+\psi$, we have that $T_{k G}^{c}\left(\phi \pi_{0}, \psi \pi_{1}\right)$ is injective by [Mo93, Theorem 5.3.1].

(ii) It follows from Part (i).

(iii). By [ZCZ, Lemma 2.1] $k D\left[k Q^{c}, D, r, \vec{\rho}, u\right] \cong \mathfrak{B}(D, r, \vec{\rho}, u) \# k D$ and $k G\left[k Q^{\prime c}, G, r^{\prime}\right.$, $\left.\overrightarrow{\rho^{\prime}}, u^{\prime}\right] \cong \mathfrak{B}\left(G, r^{\prime}, \overrightarrow{\rho^{\prime}}, u^{\prime}\right) \# k G$. Applying Part (ii) we complete the proof.

The relation " $\leq$ " has the transitivity, i.e.

Lemma 1.7. Assume that $G$ is a subgroup of $G^{\prime}$ and $G^{\prime}$ is a subgroup of $G^{\prime \prime}$. If $\operatorname{RSR}(G, r, \vec{\rho}, u) \leq \operatorname{RSR}\left(G^{\prime}, r^{\prime}, \overrightarrow{\rho^{\prime}}, u^{\prime}\right)$ and $\operatorname{RSR}\left(G^{\prime}, r^{\prime}, \overrightarrow{\rho^{\prime}}, u^{\prime}\right) \leq \operatorname{RSR}\left(G^{\prime \prime}, r^{\prime \prime}, \overrightarrow{\rho^{\prime \prime}}, u^{\prime \prime}\right)$, then $\operatorname{RSR}(G, r, \vec{\rho}, u) \leq \operatorname{RSR}\left(G^{\prime \prime}, r, \overrightarrow{\rho^{\prime \prime}}, u^{\prime \prime}\right)$.

Proof. Obviously, $G$ is a subgroup of $G^{\prime \prime}$ and $r \leq r^{\prime \prime}$. For any $C \in \mathcal{K}_{r}(G)$ and $i \in I_{C}(r, u)$, then $u(C)=u^{\prime}\left(C_{G^{\prime}}\right)=u^{\prime \prime}\left(C_{G^{\prime \prime}}\right)$. Let $s=u(C)$ and let $X_{C}^{(i)}, X_{C_{G^{\prime}}^{\prime}}^{\prime(i)}$ and $X^{\prime \prime}{ }_{C_{G^{\prime \prime}}}^{(i)}$ be representation spaces of $\rho_{C}^{(i)}, \rho_{C_{G^{\prime}}}^{\prime(i)}$ and $\rho^{\prime \prime}{ }_{C_{G^{\prime \prime}}}^{(i)}$, respectively. Let $\left(X_{C}^{(i)}, \rho_{C}^{(i)}\right)$ be isomorphic to a subrepresentation $\left(N,\left.\rho_{C_{G^{\prime}}^{\prime}}^{\prime(i)}\right|_{G^{s}}\right)$ of $\left(\left.X_{C_{G^{\prime}}^{\prime}}^{\prime(i)} \rho_{C_{G^{\prime}}}^{\prime(i)}\right|_{G^{s}}\right)$. Considering $\left(X_{C_{G^{\prime}}^{\prime}}^{\prime(i)}, \rho_{C_{G^{\prime}}^{\prime}}^{\prime(i)}\right)$ is isomorphic to a subrepresentation of $\left(X_{C_{G^{\prime \prime}}^{\prime \prime}}^{(i)},\left.\rho_{C_{G^{\prime \prime}}^{\prime \prime}}^{(i)}\right|_{G^{\prime s}}\right)$, we have that $\left(N,\left.\rho^{\prime(i)} C_{G^{\prime}}\right|_{G^{s}}\right)$ is isomorphic to a subrepresentation of $\left(X^{\prime \prime}{ }_{C_{G^{\prime \prime}}}^{(i)},\left.\rho^{\prime \prime}{ }_{C_{G^{\prime \prime}}}^{(i)}\right|_{G^{s}}\right)$. Consequently, $\left(X_{C}^{(i)}, \rho_{C}^{(i)}\right)$ is isomorphic to a subrepresentation of $\left(\left.X_{C_{G^{\prime \prime}}^{\prime \prime}}^{\prime(i)} \rho_{C_{G^{\prime \prime}}}\right|_{G^{s}}\right)$.

Lemma 1.8. Let $N$ be a subgroup of $G$ and $(X, \rho)$ be an irreducible representation of $N^{\sigma}$ with $\sigma \in N$. If the induced representation $\rho^{\prime}:=\rho \uparrow_{N^{\sigma}}^{G^{\sigma}}$ is an irreducible representation of $G^{\sigma}$, then $\operatorname{RSR}\left(N, \mathcal{O}_{\sigma}, \rho\right) \leq \operatorname{RSR}\left(G, \mathcal{O}_{\sigma}, \rho \uparrow_{N^{\sigma}}^{G^{\sigma}}\right)$.

Proof. Since $(X, \rho) \cong\left(X \otimes_{k N^{\sigma}} 1,\left.\rho^{\prime}\right|_{k N^{\sigma}}\right)$ by sending $x$ to $x \otimes 1$ for any $x \in X$, the claim holds.

Proposition 1.9. Let $G=A \rtimes D$ with $\sigma \in D, \chi \in \widehat{A^{\sigma}}, G_{\chi}=A \rtimes D_{\chi}, \rho \in \widehat{D_{\chi}^{\sigma}}$ and $\theta_{\chi, \rho}:=(\chi \otimes \rho) \uparrow G_{\chi}^{\sigma}$. Then

(i) $\operatorname{RSR}\left(G_{\chi}, \mathcal{O}_{\sigma}, \chi \otimes \rho\right) \leq \operatorname{RSR}\left(G, \mathcal{O}_{\sigma}, \theta_{\chi, \rho}\right)$

(ii) $\operatorname{RSR}\left(G_{\chi}, \mathcal{O}_{\sigma}, \chi \otimes \rho\right)$ is -1-type if and only if $\operatorname{RSR}\left(G, \mathcal{O}_{\sigma}, \theta_{\chi, \rho}\right)$ is -1-type.

(iii) $\operatorname{RSR}\left(D_{\chi}, \mathcal{O}_{\sigma}, \rho\right) \leq \operatorname{RSR}\left(G, \mathcal{O}_{\sigma}, \theta_{\chi, \rho}\right)$.

(iv) $\operatorname{RSR}\left(D_{\chi}, \mathcal{O}_{\sigma}, \rho\right)$ is -1-type if and only if $\operatorname{RSR}\left(G, \mathcal{O}_{\sigma}, \theta_{\chi, \rho}\right)$ is -1-type.

Proof. (i) It follows from Lemma 1.8.

(ii) Let $P$ and $X$ be representation spaces of $\chi$ and $\rho$, respectively. Then $(P \otimes X) \otimes_{k G_{\chi}^{\sigma}}$ $k G^{\sigma}$ is a representation space of $\rho^{\prime}:=\theta_{\chi, \rho}$. If $\rho(\sigma)=-i d$, then for any $g \in G^{\sigma}, x \in X$, $p \in P$, we have $\left((p \otimes x) \otimes_{k G_{\chi}^{\sigma}} g\right) \cdot \sigma=((p \otimes x) \cdot \sigma) \otimes_{k G_{\chi}^{\sigma}} g=-(p \otimes x) \otimes_{k G_{\chi}^{\sigma}} g$. Therefore $\rho^{\prime}(\sigma)=-i d$. Conversely, if $\rho^{\prime}(\sigma)=-i d$, then $\left((p \otimes x) \otimes_{k G_{\chi}^{\sigma}} 1\right) \cdot \sigma=((p \otimes x) \cdot \sigma) \otimes_{k G_{\chi}^{\sigma}} 1=$ $-(p \otimes x) \otimes_{k G_{\chi}^{\sigma}} 1$. Therefore $(p \otimes x) \cdot \sigma=-p \otimes x$ for any $x \in X$. 
(iii) By (i), it is enough to show $\operatorname{RSR}\left(D_{\chi}, \mathcal{O}_{\sigma}, \rho\right) \leq \operatorname{RSR}\left(G_{\chi}, \mathcal{O}_{\sigma}, \chi \otimes \rho\right)$. Let $P$ and $X$ be the representation spaces of $\chi$ and $\rho$ on $A^{\sigma}$ and $D_{\chi}^{\sigma}$, respectively. Thus $(P \otimes X, \chi \otimes \rho)$ is an irreducible representation of $G_{\chi}^{\sigma}:=A^{\sigma} \rtimes\left(D^{\sigma}\right)_{\chi}$. Considering Definition 1.4 we only need to show that $\rho$ is isomorphic to a submodule of the restriction of $\chi \otimes \rho$ on $D_{\chi}^{\sigma}$. Fix a nonzero $p \in P$ and define a map $\psi$ from $X$ to $P \otimes X$ by sending $x$ to $p \otimes x$ for any $x \in X$. It is clear that $\psi$ is a $k D_{\chi}^{\sigma}$-module isomorphism.

(iv) Considering Part (ii), we only show that $\operatorname{RSR}\left(D_{\chi}, \mathcal{O}_{\sigma}, \rho\right)$ is -1-type if and only if $\operatorname{RSR}\left(G_{\chi}, \mathcal{O}_{\sigma}, \chi \otimes \rho\right)$ is -1-type. Since $\chi_{\rho}(\sigma)=\chi_{(\chi \otimes \rho)}(\sigma)$, the claim holds.

If $D_{\chi}=D$, then it follows from the proposition above that $\operatorname{RSR}\left(D, \mathcal{O}_{\sigma}, \rho\right) \leq \operatorname{RSR}\left(G, \mathcal{O}_{\sigma}\right.$, $\left.\theta_{\chi, \rho}\right)$. Therefore we have

Corollary 1.10. Let $G=A \rtimes D$. If $r \leq r^{\prime}$ and $\rho_{C_{G}^{\prime}}^{(i)}=\theta_{\chi_{C}^{(i)}, \rho_{C}^{(i)}}$ with $D_{\chi_{C}^{(i)}}=D$, $\chi_{C}^{(i)} \in \widehat{A^{u(C)}}, u(C)=u^{\prime}\left(C_{G}\right)$ and $I_{C}(r, u) \subseteq I_{C_{G}}\left(r^{\prime}, u^{\prime}\right)$ for any $i \in I_{C}(r, u), C \in \mathcal{K}_{r}(D)$, then $\operatorname{RSR}(D, r, \vec{\rho}, u) \leq \operatorname{RSR}\left(G, r^{\prime}, \overrightarrow{\rho^{\prime}}, u^{\prime}\right)$. Furthermore, if $I_{C}(r, u)=I_{C_{G}}\left(r^{\prime}, u^{\prime}\right)$ for any $C \in \mathcal{K}_{r}(D)$ and $\mathcal{K}_{r^{\prime}}(G)=\left\{C_{G} \mid C \in \mathcal{K}_{r}(D)\right\}$, then $\operatorname{RSR}(D, r, \vec{\rho}, u)$ is -1-type if and only if $\operatorname{RSR}\left(G, r^{\prime}, \overrightarrow{\rho^{\prime}}, u^{\prime}\right)$ is -1-type.

Lemma 1.11. Let $G=G_{1} \times G_{2}$. If $\sigma=\left(\sigma_{1}, \sigma_{2}\right) \in G$ with $\rho_{1} \in \widehat{G_{1}^{\sigma_{1}}}$ and $\rho_{2} \in \widehat{G_{2}^{\sigma_{2}}}$, then

(i) $G^{\sigma}=G_{1}^{\sigma} \times G_{2}^{\sigma} ; G_{1}^{\sigma}=G_{1}^{\sigma_{1}}$ and $G_{2}^{\sigma}=G_{2}^{\sigma_{2}}$.

(ii) $\mathcal{O}_{\sigma}^{G}=\mathcal{O}_{\sigma_{1}}^{G_{1}} \times \mathcal{O}_{\sigma_{2}}^{G_{2}}$, where $\mathcal{O}_{\sigma}^{G}$ denotes the conjugacy class containing $\sigma$ of $G$.

(iii) $\operatorname{RSR}\left(G_{1}, \mathcal{O}_{\sigma_{1}}, \rho_{1}\right) \leq \operatorname{RSR}\left(G, \mathcal{O}_{\sigma_{1}}, \rho_{1} \otimes \rho_{2}\right)$ when $\sigma_{2}=1 ; \operatorname{RSR}\left(G_{2}, \mathcal{O}_{\sigma_{2}}, \rho_{2}\right) \leq$ $\operatorname{RSR}\left(G, \mathcal{O}_{\sigma_{2}}, \rho_{1} \otimes \rho_{2}\right)$ when $\sigma_{1}=1$.

Proof. (i) It is clear $G_{1}^{\sigma}=G_{1}^{\sigma_{1}}$ and $G_{2}^{\sigma}=G_{2}^{\sigma_{2}}$. For any $x=(a, h) \in G^{\sigma}$, then $x \sigma=\sigma x$, which implies that $a \sigma_{1}=\sigma_{1} a$ and $h \sigma_{2}=\sigma_{2} h$. Thus $x \in G_{1}^{\sigma} \times G_{2}^{\sigma}$ and $G^{\sigma} \subseteq G_{1}^{\sigma} \times G_{2}^{\sigma}$. Similarly, we have $G_{1}^{\sigma} \times G_{2}^{\sigma} \subseteq G^{\sigma}$.

(ii) It is clear.

(iii) We only show the first claim. It is clear that $\rho_{1}$ is isomorphic to a subrepresentation of the restriction of $\rho_{1} \otimes \rho_{2}$ on the $G_{1}^{\sigma_{1}}$. Indeed, assume that $X$ and $Y$ are the representation spaces of $\rho_{1}$ and $\rho_{2}$, respectively. Obviously, $G_{1}^{\sigma_{1}}$-module $\left(X, \rho_{1}\right)$ is isomorphic to a submodule of the restriction of $\rho_{1} \otimes \rho_{2}$ on $G_{1}^{\sigma_{1}}$ under isomorphism $\psi$ form $X$ to $X \otimes y_{0}$ by sending $x$ to $x \otimes y_{0}$ for any $x \in X$, where $y_{0}$ is a non-zero fixed element in $Y$.

Lemma 1.12. Let $G=G_{1} \times G_{2}$ and $\sigma=\left(\sigma_{1}, \sigma_{2}\right) \in G$ with $\rho_{1} \in \widehat{G_{1}^{\sigma_{1}}}$ and $\rho_{2} \in \widehat{G_{2}^{\sigma_{2}}}$.

(i) If $\sigma_{2}=1$, then $\operatorname{RSR}\left(G, \mathcal{O}_{\sigma_{1}}, \rho_{1} \otimes \rho_{2}\right)$ is -1-type if and only if $\operatorname{RSR}\left(G_{1}, \mathcal{O}_{\sigma_{1}}, \rho_{1}\right)$ is -1-type.

(ii) If $\sigma_{1}=1$, then $\operatorname{RSR}\left(G, \mathcal{O}_{\sigma_{2}}, \rho_{1} \otimes \rho_{2}\right)$ is -1-type if and only if $\operatorname{RSR}\left(G_{2}, \mathcal{O}_{\sigma_{2}}, \rho_{2}\right)$ is -1-type.

Proof. (i) Considering $\chi_{\rho_{1} \otimes \rho_{2}}\left(\sigma_{1}\right)=\chi_{\rho_{1}}\left(\sigma_{1}\right) \operatorname{deg}\left(\rho_{2}\right)$, we can complete the proof.

(ii) It is similar. 
Lemma 1.13. $\theta_{\chi, \rho}$ is a one dimensional representation of $G^{\sigma}=A^{\sigma} \rtimes D^{\sigma}$ if and only if $D_{\chi}^{\sigma}=D^{\sigma}$ and $\operatorname{deg} \rho=1$

Proof. Let $P$ and $X$ be the representation spaces of $\chi$ and $\rho$ on $A^{\sigma}$ and $D_{\chi}^{\sigma}$, respectively. $\left((P \otimes X) \otimes_{k G_{\chi}^{\sigma}} k G^{\sigma}, \theta_{\chi, \rho}\right)$ is a one dimensional representation of $G^{\sigma}=A^{\sigma} \rtimes D^{\sigma}$ if and only if $k G^{\sigma}=k G_{\chi}^{\sigma}$ and $\operatorname{dim} X=1$. However. $k G^{\sigma}=k G_{\chi}^{\sigma}$ if and only if $D_{\chi}^{\sigma}=D^{\sigma}$.

Consequently, $\theta_{\chi, \rho}=\chi \otimes \rho$ when $\theta_{\chi, \rho}$ is one dimensional representation.

\section{Symmetric GROUP $\mathbb{S}_{n}$}

In this section we study the Nichols algebras over symmetric groups.

Without specification, $\sigma \in \mathbb{S}_{n}$ is always of type $1^{\lambda_{1}} 2^{\lambda_{2}} \cdots n^{\lambda_{n}} \cdot g_{j}$ denotes the generator of cycle group $C_{j}$ with order $j$ for natural number $j$. We keep on the work in [Su78, Page 295-299]. Let $r_{j}:=\sum_{1 \leq k \leq j-1} k \lambda_{k}$ and $\sigma_{j}:=\prod_{1 \leq l \leq \lambda_{j}}\left(y_{r_{j}+(l-1) j+1}\right.$, $\left.y_{r_{j}+(l-1) j+2}, \quad \cdots, \quad y_{r_{j}+l j}\right)$, the multiplication of cycles of length $j$ in the independent cycle decomposition of $\sigma$, as well as $Y_{j}:=\left\{y_{s} \mid s=r_{j}+1, \cdots, r_{j+1}\right\}$. Therefore $\sigma=\prod \sigma_{i}$ and $\left(\mathbb{S}_{n}\right)^{\sigma}=\prod\left(\mathbb{S}_{Y_{i}}\right)^{\sigma_{i}}=T_{1} \times \cdots \times T_{n}$. It follows from [AFZ, subsection 2.2] that $T_{j}$ is generated by $A_{1, j}, \ldots, A_{\lambda_{j}, j}, B_{1, j}, \ldots, B_{\lambda_{j}-1, j}$, where $A_{1,1}=\left(y_{1}\right), \ldots, A_{\lambda_{1}, 1}=\left(y_{\lambda_{1}}\right)$, $A_{1,2}=\left(\begin{array}{ll}y_{\lambda_{1}+1} & y_{\lambda_{1}+2}\end{array}\right), \ldots, A_{\lambda_{2}, 2}=\left(\begin{array}{ll}y_{\lambda_{1}+2 \lambda_{2}-1} & y_{\lambda_{1}+2 \lambda_{2}}\end{array}\right)$, and so on. More precisely, if $1<j \leq n$, then

$$
\begin{aligned}
& A_{l, j}:=\left(y_{r_{j}+(l-1) j+1}, \quad y_{r_{j}+(l-1) j+2}, \quad \cdots, \quad y_{r_{j}+l j}\right), \\
& B_{h, j}:=\left(y_{r_{j}+(h-1) j+1}, \quad y_{r_{j}+h j+1},\right)\left(y_{r_{j}+(h-1) j+2}, \quad y_{r_{j}+h j+2}\right) \cdots\left(y_{r_{j}+h j}, \quad y_{r_{j}+(h+1) j}\right),
\end{aligned}
$$

for all $l, h$, with $1 \leq l \leq \lambda_{j}, 1 \leq h \leq \lambda_{j}-1$. Notice that $\varphi\left(A_{l, j}\right)=(\overbrace{\left(1, \cdots, 1, g_{j}^{j-1}\right.}^{l}$ $, 1 \cdots, 1), 1)$ and $\varphi\left(B_{h, j}\right)=(1,(h, h+1))$, where $\varphi$ is an isomorphism from $G^{\sigma_{j}}$ to $\left(C_{j}\right)^{\lambda_{j}} \rtimes \mathbb{S}_{\lambda_{j}}$, defined in the proof of [ZWW, Pro. 2.10] (also see the below (2.1)). Furthermore, if $\cup_{i>1} Y_{i} \subseteq X \subseteq\{1,2, \cdots, n\}$, then $\sigma$ is said to be in $\mathbb{S}_{X}$.

Lemma 2.1. If $\sigma \in \mathbb{S}_{n}$ is the multiplication of $m$ independent cycles with the same length $l$, i.e. $\sigma$ is of type $l^{m}$, then

(i) $\varphi(\sigma)=\left(\left(g_{l}^{l-1}, g_{l}^{l-1}, \cdots, g_{l}^{l-1}\right),(1)\right)$, where $\varphi$ is the isomorphism from $\left(\mathbb{S}_{n}\right)^{\sigma}$ to $\left(C_{l}\right)^{m} \rtimes \mathbb{S}_{m}$ defined in the proof of [ZWW, Pro. 2.10].

(ii) $\theta_{\chi, \rho}(\varphi(\sigma))=\chi\left(\left(g_{l}^{l-1}, g_{l}^{l-1}, \cdots, g_{l}^{l-1}\right)\right)$ id for any $\rho \in \widehat{\left(\mathbb{S}_{m}\right)_{\chi}}$ and $\chi \in \widehat{\left(C_{l}\right)^{m}}$, where $G=\left(C_{l}\right)^{m} \rtimes \mathbb{S}_{m}, G_{\chi}=\left(C_{l}\right)^{m} \rtimes\left(S_{m}\right)_{\chi}$ and $\theta_{\chi, \rho}=(\chi \otimes \rho) \uparrow_{G_{\chi}}^{G}$.

Proof. (i) Assume that

$$
\sigma=\left(a_{10} a_{11} \cdots a_{1, l-1}\right)\left(a_{20} \cdots a_{2, l-1}\right) \cdots\left(a_{m 0} \cdots a_{m, l-1}\right) .
$$

By [ZWW, Pro. 2.14] or [Su78],

$$
\mathbb{S}_{n}^{\sigma} \stackrel{\varphi}{\cong}\left(C_{l}\right)^{m} \rtimes S_{m}
$$


where the map $\varphi$ is the same as in the proof of [ZWW, Pro. 2.10 ]. Indeed, here is precise definition of isomorphism $\varphi$. For any element $\tau$ of $\left(\mathbb{S}_{n}\right)^{\sigma}$, we will define $\theta(\tau) \in S_{m}$ and $f_{\tau} \in\left(C_{l}\right)^{m}$ by

$$
\tau^{-1}\left(a_{i 0}\right)=a_{j k}, \quad j=\theta(\tau)^{-1}(i), \quad f_{\tau}(i)=g_{l}^{k},
$$

where $g_{l}$ is the generator of $C_{l}, 1 \leq i \leq m$. Let

$$
\varphi(\tau)=\left(f_{\tau}, \theta(\tau)\right) .
$$

Since $\sigma\left(a_{i, l-1}\right)=a_{i, 0}$, we have $\varphi(\sigma)=\left(f_{\sigma}, \theta(\sigma)\right)$ with $f_{\sigma}=\left(g_{l}^{l-1}, g_{l}^{l-1}, \cdots, g_{l}^{l-1}\right) \in\left(C_{l}\right)^{m}$ and $\theta(\sigma)=(1) \in \mathbb{S}_{m}$.

(ii) Let $P$ and $X$ be representation spaces of $\chi$ and $\rho$, respectively. For any $0 \neq p \in P$ and $0 \neq x \in X$, see

$$
\begin{aligned}
\left((p \otimes x) \otimes_{k G_{\chi}} 1\right) \cdot \varphi(\sigma) & =((p \otimes x) \cdot \varphi(\sigma)) \otimes_{k G_{\chi}} 1 \\
& \left.=\chi\left(\left(g_{l}^{l-1}, g_{l}^{l-1}, \cdots, g_{l}^{l-1}\right)\right)\left((p \otimes x) \otimes_{k G_{\chi}} 1\right) \quad \text { (by Part (i) }\right) .
\end{aligned}
$$

Since $\varphi(\sigma)$ is in the center of $C_{l}^{m} \rtimes \mathbb{S}_{m}$ and $\theta_{\chi, \rho}$ is irreducible, we have that Part (ii) holds.

Obviously, every element in $\widehat{\left(C_{l}\right)^{m}}$ can be denoted by $\chi_{\left(t_{1, l}, t_{2, l}, \cdots, t_{m, l} ; l\right)}:=\chi_{l}^{t_{1, l}} \otimes \chi_{l}^{t_{2, l}} \otimes$ $\cdots \otimes \chi_{l}^{t_{m, l}}$ for $0 \leq t_{j, l} \leq l-1$. For convenience, we denote $\chi_{\left(t_{1, l}, t_{2, l}, \cdots, t_{m, l} ; l\right)}$ by $\chi^{\mathrm{t}}$ when it does not cause mistake.

Lemma 2.2. (i) Every irreducible representation of $\mathbb{S}_{n}^{\sigma}=\prod_{1 \leq i \leq n} \mathbb{S}_{Y_{i}}^{\sigma_{i}}$ is isomorphic to one of the following list:

$$
\otimes_{1 \leq i \leq n}\left(\theta_{\chi^{\mathrm{t}_{i}, \rho_{i}}} \varphi_{i}\right)
$$

where $\rho_{i} \in\left(\widehat{\left.\mathbb{S}_{Y_{i}}\right)_{\chi^{t_{i}}}}\right.$ and $\varphi_{i}$ is the isomorphism from $\mathbb{S}_{Y_{i}}^{\sigma_{i}}$ to $\left(C_{i}\right)^{\lambda_{i}} \rtimes \mathbb{S}_{\lambda_{i}}$ as in (2.1).

(ii) Let $\chi$ denote the character of $\otimes_{1 \leq i \leq n}\left(\theta_{\chi^{t_{i}, \rho_{i}}} \varphi_{i}\right)$. Then

$$
\chi(\sigma)=\prod_{1 \leq i \leq n}\left(\prod_{1 \leq j \leq \lambda_{i}} \chi_{i}\left(g_{i}\right)^{(i-1) t_{j, i}}\right) \operatorname{deg}\left(\theta_{\chi^{t_{i}, \rho_{i}}} \varphi_{i}\right) .
$$

Proof. (i) By [ZWW, Pro. 2.10], $\mathbb{S}_{n}^{\sigma}=\prod_{1 \leq i \leq n} \mathbb{S}_{Y_{i}}^{\sigma_{i}} \stackrel{\prod_{\varphi} \varphi_{i}}{\cong} \prod_{1 \leq i \leq n}\left(C_{i}\right)^{\lambda_{i}} \rtimes \mathbb{S}_{\lambda_{i}}$. It follows from [Se, Pro.25] that every irreducible representation of $\left(C_{i}\right)^{\lambda_{i}} \rtimes \mathbb{S}_{\lambda_{i}}$ is isomorphic to $\theta_{\chi^{t_{i}, \rho_{i}}}$. This completes our proof.

(ii) It follows from (i) and Lemma 2.1,

\section{Definition 2.3.}

$$
\xi_{\mathbf{t}, \sigma}:=\sum_{1 \leq k \leq n, 1 \leq j \leq \lambda_{k}} \frac{t_{j, k}}{k}+\frac{1}{2}
$$

is called the distinguished element of the representation (2.2) or $\operatorname{RSR}\left(\mathbb{S}_{n}, \mathcal{O}_{\sigma}, \otimes_{1 \leq i \leq n}\left(\theta_{\chi^{t_{i}, \rho_{i}}} \varphi_{i}\right)\right)$, where $0 \leq t_{j, k} \leq k-1$. 
Lemma 2.4. Let $\chi$ denote the character of $\left(\otimes_{1 \leq k \leq n}\left(\theta_{\chi^{t_{k}, \rho_{k}}} \varphi_{k}\right)\right)$. Then $\xi_{\mathbf{t}, \sigma}$ is an integer if and only if $\chi(\sigma)=-\operatorname{deg}(\chi)$.

Proof. Let $\omega_{j}:=e^{\frac{2 \pi i}{j}}$, where $i:=\sqrt{-1}$ and $e$ is the Euler's constant. For any $k$ with $\lambda_{k} \neq 0$, since $(k, k-1)=1$, there exists $a_{k}$ with $\left(a_{k}, k\right)=1$ such that $\omega_{k}^{a_{k}(k-1)}=\omega_{k}$. Choice $\chi_{k}$ such that $\chi_{k}\left(g_{k}\right)=\omega_{k}^{a_{k}}$. By formula (2.3), we have

$$
\chi(\sigma)=e^{(2 \pi i) \sum_{1 \leq k \leq n, 1 \leq j \leq \lambda_{k}} \frac{t_{j, k}}{k}} \operatorname{deg}\left(\otimes_{1 \leq s \leq n}\left(\theta_{\chi^{t_{s}, \rho_{s}}} \varphi_{s}\right)\right) .
$$

Using the formula we complete the proof.

Consequently, we have

Proposition 2.5. The distinguished element $\xi_{\mathbf{t}, \sigma}$ of the representation $\otimes_{1 \leq i \leq n}\left(\theta_{\chi^{t_{i}, \rho_{i}}} \varphi_{i}\right)$, as in (2.2) is an integer and the order of $\sigma$ is even if and only if $\mathfrak{B}\left(, \mathcal{O}_{\sigma}^{\mathbb{S}_{n}}, \otimes_{1 \leq i \leq n}\left(\theta_{\chi^{t_{i}, \rho_{i}}} \varphi_{i}\right)\right)$ is -1-type.

Lemma 2.6. If $\chi \in \widehat{\left(C_{l}\right)^{m}}$, then $\left(\mathbb{S}_{m}\right)_{\chi}=\mathbb{S}_{m}$ if and only if $\chi=\chi_{l}^{t} \otimes \chi_{l}^{t} \otimes \cdots \otimes \chi_{l}^{t}$ for some $0 \leq t \leq l-1$.

Proof. Note that $\mathbb{S}_{m}$ acts $\left(C_{l}\right)^{m}$ as follows: For any $a \in C_{l}^{m}$ with $a=\left(g_{l}^{a_{1}}, g_{l}^{a_{2}}, \cdots, g_{l}^{a_{m}}\right)$ and $h \in \mathbb{S}_{m}$,

$$
h \cdot a=\left(g_{l}^{a_{h^{-1}(1)}}, g_{l}^{a_{h^{-1}(2)}}, \cdots, g_{l}^{a_{h^{-1}(m)}}\right) .
$$

Let $\chi=\otimes_{i=1}^{m} \chi_{l}^{t_{i}}$ with $0 \leq t_{i} \leq l-1$. If $\left(\mathbb{S}_{m}\right)_{\chi}=\mathbb{S}_{m}$ and there exist $i \neq j$ such that $t_{i} \neq t_{j}$. Set $a=\left(g_{l}^{a_{1}}, g_{l}^{a_{2}}, \cdots, g_{l}^{a_{m}}\right) \in C_{l}^{m}$ with $a_{i}=1$ and $a_{s}=0$ when $s \neq i ; h=(i, j) \in \mathbb{S}_{m}$. See

$$
\begin{aligned}
(h \cdot \chi)(a) & =\chi\left(h^{-1} \cdot a\right)=\chi\left(g_{l}^{a_{h(1)}}, g_{l}^{a_{h(2)}}, \cdots, g_{l}^{a_{h(m)}}\right) \\
& =\chi_{l}\left(g_{l}\right)^{t_{i} a_{j}+t_{j} a_{i}}=\chi_{l}\left(g_{l}\right)^{t_{j}}
\end{aligned}
$$

and $\chi(a)=\chi_{l}\left(g_{l}\right)^{t_{i}}$. This implies $\chi \neq(h \cdot \chi)$. We get a contradiction. Conversely, it is clear.

Proposition 2.7. Every one dimensional representation of $\mathbb{S}_{n}^{\sigma}$ is of the following form:

$$
\otimes_{1 \leq i \leq n}\left(\chi_{\left(t_{i}, \cdots, t_{i} ; i\right)} \otimes \rho_{i}\right) \varphi_{i},
$$

where $1 \leq t_{i} \leq i-1$ and $\rho_{i}$ is a one dimensional representation for any $1 \leq i \leq n$.

Proof. By Lemma 2.2, every irreducible representation of $\mathbb{S}_{n}^{\sigma}$ is of form as (2.2). If (2.2) is one dimensional, then it follows from Lemma 1.13 that $\otimes_{1 \leq i \leq n}\left(\theta_{\chi^{t_{i}, \rho_{i}}} \varphi_{i}\right)=$ $\otimes_{1 \leq i \leq n}\left(\chi^{\mathbf{t}_{i}} \otimes \rho_{i}\right) \varphi_{i}$ and $\left(\mathbb{S}_{\lambda_{i}}\right)_{\chi^{\mathrm{t}_{i}}}=\mathbb{S}_{\lambda_{i}}$. By Lemma2.5, $\chi^{\mathbf{t}_{i}}=\chi_{i}^{t_{i}} \otimes \chi_{i}^{t_{i}} \otimes \cdots \otimes \chi_{i}^{t_{i}}=\chi_{\left(t_{i}, \cdots, t_{i} ; i\right)}$ for some $0 \leq t_{i} \leq i-1$.

Note that every one dimensional representation of $\mathbb{S}_{m}$ is $\chi_{2}$ or $\epsilon$. Therefore every one dimensional representation of $\mathbb{S}_{m}^{\sigma}$ can be denoted by $\otimes_{1 \leq i \leq n}\left(\chi_{\left(t_{i}, \cdots, t_{i} ; i\right)} \otimes \chi_{2}^{\delta_{i}}\right)$ in short, where $\delta_{i}=1$ or 0 . 
Consequently, the distinguished element of one dimensional representation (2.7) becomes

$$
\xi_{\mathbf{t}, \sigma}=\sum_{1 \leq k \leq n} \frac{t_{k} \lambda_{k}}{k}+\frac{1}{2} \text { with } 0 \leq t_{k} \leq k-1
$$

For any $\mu=\otimes_{1 \leq i \leq n}\left(\theta_{\chi^{\mathrm{t}_{i}, \rho_{i}}} \varphi_{i}\right) \in \widehat{\mathbb{S}_{n}^{\sigma}}$ as Lemma 2.2, let $\mu_{j}:=\theta_{\chi^{\mathrm{t}_{i}, \rho_{i}}} \varphi_{i}$ be a representation of $\mathbb{S}_{Y_{i}}^{\sigma_{i}}$ and $\mu=\prod_{1 \leq j \leq n} \mu_{j}$. We often omit $\varphi_{i}$.

Proposition 2.8. Let $\sigma \in \mathbb{S}_{n}$ be of type $\left(1^{\lambda_{1}}, 2^{\lambda_{2}}, \ldots, n^{\lambda_{n}}\right)$ and $\mu=\otimes_{1 \leq i \leq n} \mu_{i}$ with $\mu_{i}:=\left(\theta_{\chi^{\mathbf{t}_{i}, \rho_{i}}} \varphi_{i}\right)$ as in (2.2). Then $\mathfrak{B}\left(\mathcal{O}_{\sigma}^{\mathbb{S}_{n}}, \mu\right)$ is -1-type in the following cases.

(i) $\left(1^{\lambda_{1}}, 2\right), \mu_{1}=\operatorname{sgn}$ or $\epsilon, \mu_{2}=\chi_{(1 ; 2)}$.

(ii) $\left(2, \sigma_{o}\right), \sigma_{o}:=\prod_{1<i<n, 1<i \text { is odd }} \sigma_{i} \neq \mathrm{id}, \mu_{2}=\chi_{(1 ; 2)}, \mu_{j}=\left(\chi_{(0, \ldots, 0 ; j)} \otimes \rho_{j}\right) \uparrow_{\left(\mathbb{S}_{Y_{j}}\right)_{\chi_{(0, \ldots, 0 ; j)}}^{\sigma_{j}}}^{\left(\mathbb{S}_{Y_{j}}\right)^{\sigma_{j}}}$, for all odd $j>1$.

(iii) $\left(1^{\lambda_{1}}, 2^{3}\right), \mu_{1}=\operatorname{sgn}$ or $\epsilon, \mu_{2}=\chi_{(1,1,1 ; 2)} \otimes \epsilon$ or $\chi_{(1,1,1 ; 2)} \otimes \operatorname{sgn}$.

Furthermore, if $\lambda_{1}>0$, then $\mu_{2}=\chi_{(1,1,1 ; 2)} \otimes \operatorname{sgn}$.

(iv) $\left(2^{5}\right), \mu_{2}=\chi_{(1,1,1,1,1 ; 2)} \otimes \epsilon$ or $\chi_{(1,1,1,1,1 ; 2)} \otimes \operatorname{sgn}$.

(v) $\left(1^{\lambda_{1}}, 4\right), \mu_{1}=\operatorname{sgn}$ or $\epsilon, \mu_{4}=\chi_{(2 ; 4)}$.

(vi) $\left(1^{\lambda_{1}}, 4^{2}\right), \mu_{1}=\operatorname{sgn}$ or $\epsilon, \mu_{4}=\chi_{(1,1 ; 4)} \otimes \operatorname{sgn}$ or $\chi_{(3,3 ; 4)} \otimes \operatorname{sgn}$.

(vii) $(2,4), \mu_{2}=\chi_{(1 ; 2)}$ and $\mu_{4}=\epsilon$ or $\mu_{2}=\epsilon$ and $\mu_{4}=\chi_{(2 ; 4)}$.

(viii) $\left(2,4^{2}\right), \mu_{2}=\epsilon, \mu_{4}=\chi_{(1,1 ; 4)} \otimes \operatorname{sgn}$ or $\chi_{(3,3 ; 4)} \otimes \operatorname{sgn}$.

(ix) $\left(2^{2}, 4\right), \operatorname{deg} \mu_{2}=1, \mu_{4}=\chi_{(2 ; 4)}$.

Proof. It is sufficient to show that their distinguished element $\xi_{\mathbf{t}, \sigma}$, defined in Definition 2.3, is an integer by Lemma 2.4.

(i) $\xi_{\mathbf{t}, \sigma}=\frac{1}{2}+\frac{1}{2}=1$.

(ii) $\xi_{\mathbf{t}, \sigma}=\frac{1}{2}+\frac{1}{2}=1$.

(iii) $\xi_{\mathbf{t}, \sigma}=\frac{3}{2}+\frac{1}{2}=2$.

(iv) $\xi_{\mathbf{t}, \sigma}=\frac{5}{2}+\frac{1}{2}=3$.

(v) $\xi_{\mathrm{t}, \sigma}=\frac{2}{4}+\frac{1}{2}=1$.

(vi) $\xi_{\mathbf{t}, \sigma}=\frac{1}{4}+\frac{1}{4}+\frac{1}{2}=1$ or $\xi_{\mathbf{t}, \sigma}=\frac{3}{4}+\frac{3}{4}+\frac{1}{2}=2$.

(vii) $\xi_{\mathbf{t}, \sigma}=\frac{1}{2}+\frac{1}{2}=1$ or $\xi_{\mathbf{t}, \sigma}=\frac{2}{4}+\frac{1}{2}=1$.

(viii) $\xi_{\mathbf{t}, \sigma}=\frac{2}{4}+\frac{1}{2}=1$ or $\xi_{\mathbf{t}, \sigma}=\frac{6}{4}+\frac{1}{2}=2$.

(ix) Assume $\mu_{2}=\chi_{(t, t ; 2)} \otimes \rho_{2}$. Thus $\xi_{\mathbf{t}, \sigma}=\frac{2 t}{2}+\frac{2}{4}+\frac{1}{2}=1+t$.

We only show this for case (vi) since others is similar. Therefore the distinguished element $\xi_{\mathbf{t}, \sigma}$ is an integer.

In fact, it follows from [AFZ, Theorem 1] that if $\left.\operatorname{dim} \mathfrak{B O}_{\sigma}^{\mathbb{S}_{n}}, \mu\right)<\infty$ then some of the case (i)-(ix) in proposition above hold. 


\section{The classical Weyl groups}

By Ca72, $\left(C_{2}\right)^{n} \rtimes \mathbb{S}_{n}$ is isomorphic to the Weyl groups of $B_{n}$ and $C_{n}$ with $n>2$. Obviously, when $A=\left\{a \in\left(C_{2}\right)^{n} \mid a=\left(g_{2}^{a_{1}}, g_{2}^{a_{2}}, \cdots, g_{2}^{a_{n}}\right)\right.$ with all $\left.a_{i}=0\right\}, A \rtimes \mathbb{S}_{n}$ is isomorphic to the Weyl groups of $A_{n-1}$ with $n>1$. The Weyl group $W\left(D_{n}\right)$ of $D_{n}$ is a subgroup of $W\left(B_{n}\right)$, Weyl groups of $B_{n}$. Without specification, $A \subseteq\left(C_{2}\right)^{n}, \mathbb{S}_{n} \cdot A \subseteq A$ and $G=A \rtimes \mathbb{S}_{n}$ with $\sigma \in \mathbb{S}_{n}$. Note that $\mathbb{S}_{n}$ acts $A$ as follows: for any $a \in A$ with $a=\left(g_{2}^{a_{1}}, g_{2}^{a_{2}}, \cdots, g_{2}^{a_{n}}\right)$ and $h \in \mathbb{S}_{n}$, define

$$
h \cdot a:=\left(g_{2}^{a_{h^{-1}(1)}}, g_{2}^{a_{h^{-1}(2)}}, \cdots, g_{2}^{a_{h^{-1}(n)}}\right) .
$$

Let $G=A \rtimes \mathbb{S}_{n} . \quad(a, \sigma) \in G$ is called a sign cycle if $\sigma=\left(i_{1}, i_{2}, \cdots, i_{r}\right)$ is cycle and $a=\left(g_{2}^{a_{1}}, \cdots, g_{2}^{a_{n}}\right)$ with $a_{i}=0$ for $i \notin\left\{i_{1}, i_{2}, \cdots, i_{r}\right\}$. A sign cycle $(a, \sigma)$ is called positive (or negative ) if $\sum_{i=1}^{n} a_{i}$ is even (or odd). $(a, \sigma)=\left(a^{(1)}, \sigma_{1}\right)\left(a^{(2)}, \sigma_{2}\right) \cdots\left(a^{(r)}, \sigma_{r}\right)$ is called an independent sign cycle decomposition of $(a, \sigma)$ if $\sigma=\sigma_{1} \sigma_{2} \cdots \sigma_{r}$ is an independent cycle decomposition of $\sigma$ in $\mathbb{S}_{n}$ and $\left(a^{(i)}, \sigma_{i}\right)$ is a sign cycle for $1 \leq i \leq r$. Furthermore, $(a, \sigma) \in A \rtimes \mathbb{S}_{n}$ is called positive ( or negative ) if $\sum_{i=1}^{n} a_{i}$ is even (or odd). The type of $\sigma$ is said to be the type of $(a, \sigma)$.

\subsection{Match.}

Lemma 3.1. Let $\chi^{(\nu)}$ denote the one dimensional representation $\left(\otimes_{j=1}^{\nu} \chi_{2}\right) \otimes\left(\otimes_{j=\nu+1}^{n} \epsilon\right)$ $=\overbrace{\chi_{2} \otimes \cdots \otimes \chi_{2}}^{\nu} \otimes \overbrace{\epsilon \otimes \cdots \otimes \epsilon}^{n-\nu}$ of $A \subseteq\left(C_{2}\right)^{n}$ for $\nu=0,1, \cdots, n$. Then $\left\{\chi^{(\nu)} \mid \nu=\right.$ $0,1, \cdots, j\}$ be a system of the representatives for the orbits of $\mathbb{S}_{n}$ on $\hat{A}$, where $j=0, n-1, n$ when $A \rtimes \mathbb{S}_{n}$ is isomorphic to the Weyl groups of $A_{n-1}, D_{n}$, and $B_{n}$, respectively. Furthermore, $\left(\mathbb{S}_{n}\right)_{\chi^{(\nu)}}=\left\{\sigma \in \mathbb{S}_{n} \mid 1 \leq \sigma(i) \leq \nu\right.$ when $\left.1 \leq i \leq \nu\right\}=\mathbb{S}_{\{1,2, \cdots, \nu\}} \times \mathbb{S}_{\{\nu+1, \nu+2, \cdots, n\}}$ for $\nu=0,1, \cdots, n$.

Lemma 3.2. Let $1 \leq \nu \leq n-1, \sigma \in \mathbb{S}_{n}$. Then

(i) $\left(\left(\mathbb{S}_{n}\right)_{\chi^{(\nu)}}\right)^{\sigma}=\left(\left(\mathbb{S}_{n}\right)^{\sigma}\right)_{\chi^{(\nu)}}$;

(ii) $\left(\left(\mathbb{S}_{n}\right)_{\chi^{(\nu)}}\right)^{\sigma}=\mathbb{S}_{\{1,2, \cdots, \nu\}}^{\sigma} \times \mathbb{S}_{\{1+\nu, 2+\nu, \cdots, n\}}^{\sigma}$ when $\sigma \in\left(\mathbb{S}_{n}\right)_{\chi^{(\nu)}}$.

(iii) If $\chi^{(\nu)} \in \widehat{A^{\sigma}}$ then $\sigma \in\left(\mathbb{S}_{n}\right)_{\chi^{(\nu)}}$.

Proof. (i) It is clear. (ii) It follows from Lemma 1.11. (iii) Since $\chi^{(\nu)} \in \widehat{A^{\sigma}},\left(\sigma \cdot \chi^{(\nu)}\right)(a)$ $=\chi^{(\nu)}\left(\sigma^{-1} a \sigma\right)=\chi^{(\nu)}(a)$ for any $a \in A^{\sigma}$. Therefore $\sigma \cdot \chi^{(\nu)}=\chi^{(\nu)}$, i.e. $\sigma \in\left(\mathbb{S}_{n}\right)_{\chi^{(\nu)}}$.

Definition 3.3. Let $G=A \rtimes \mathbb{S}_{n}$. If $\sigma \in \mathbb{S}_{\nu}$ or $\sigma \in \mathbb{S}_{\{\nu+1, \cdots, n\}}$, then $\operatorname{RSR}\left(G, \mathcal{O}_{\sigma}, \theta_{\chi^{(\nu)}, \rho}\right)$ is called to be matched and the distinguished element of $\operatorname{RSR}\left(\mathbb{S}_{\nu}, \mathcal{O}_{\sigma}, \rho\right)$ (when $\sigma \in \mathbb{S}_{\nu}$ ) or $\operatorname{RSR}\left(\mathbb{S}_{\{\nu+1, \cdots, n\}}, \mathcal{O}_{\sigma}, \rho\right)$ (when $\left.\sigma \in \mathbb{S}_{\{\nu+1, \cdots, n\}}\right)$ is called the distinguished element of $\operatorname{RSR}\left(G, \mathcal{O}_{\sigma}, \theta_{\chi, \rho}\right)$.

Lemma 3.4. Let $D$ be a subgroup of group $G$ and $(X, \rho)$ a representation of $D$. If $\psi$ is a group isomorphism from $G$ to $G^{\prime}$, then $\rho \uparrow_{D}^{G}=\left(\left(\rho \psi^{-1}\right) \uparrow_{\psi(D)}^{\psi(G)}\right) \psi$. 
Proof. let left coset decompositions of $D$ in $G$ be $D=\bigcup_{i=1}^{n} t_{i} D$. Then $\psi(D)=$ $\bigcup_{i=1}^{n} \psi\left(t_{i}\right) \psi(D)$ is a left coset decompositions of $\psi(D)$ in $G^{\prime}$. For any $g \in D$, by [Sa01, Definition 1.12.2], $\left(\rho \uparrow_{D}^{G}\right)(g)=\left(\rho\left(t_{i}^{-1} g t_{j}\right)\right)_{n \times n}$ and $\left(\left(\rho \psi^{-1}\right) \uparrow_{\psi(D)}^{\psi(G)}\right) \psi(g)=\left(\rho\left(t_{i}^{-1} g t_{j}\right)\right)_{n \times n}$. Thus $\rho \uparrow_{D}^{G}=\left(\left(\rho \psi^{-1}\right) \uparrow_{\psi(D)}^{\psi(G)}\right) \psi$.

Lemma 3.5. Let $G:=A \rtimes \mathbb{S}_{n}$. If $\sigma \in \mathbb{S}_{n}$ and $\theta_{\chi, \rho} \in \widehat{G^{\sigma}}$, then there exist a natural number $\nu, 0 \leq \nu \leq n, \sigma^{\prime} \in \mathbb{S}_{n}$ and $\rho^{\prime} \in \widehat{\left(\mathbb{S}_{n}\right)_{\chi^{\prime}}^{\sigma^{\prime}}}$ such that $\operatorname{RSR}\left(G, \mathcal{O}_{\sigma}, \theta_{\chi, \rho}\right) \cong$ $\operatorname{RSR}\left(G, \mathcal{O}_{\sigma^{\prime}}, \theta_{\chi^{(\nu)}, \rho^{\prime}}\right)$.

Proof. We show this by following several steps.

(i) Since $\chi \in \widehat{A^{\sigma}}$, then there exists a $W \subseteq\{1,2, \cdots, n\}$ such that $\chi=\otimes_{i=1}^{n} \chi_{2}^{\delta_{i}}$ with $\delta_{i}=1$ when $i \in W$ and $\delta_{i}=0$ otherwise.

(ii) Let $\nu=|W|$. Let $\phi \in \mathbb{S}_{n}$ with $\phi(W)=\{1,2, \cdots, \nu\}$ and $\sigma^{\prime}=\phi \sigma \phi^{-1}$. Define a map $\psi$ from $\left(\mathbb{S}_{n}\right)^{\sigma}$ to $\left(\mathbb{S}_{n}\right)^{\sigma^{\prime}}$ by sending $x$ to $\phi x \phi^{-1}$ for any $x \in\left(\mathbb{S}_{n}\right)^{\sigma}$. Let $\beta$ be a bijective map from $A^{\sigma}$ to $A^{\psi(\sigma)}$ by sending $a=\left(g_{2}^{a_{1}}, g_{2}^{a_{2}}, \cdots, g_{2}^{a_{n}}\right)$ to $b=\left(g_{2}^{a_{\phi^{-1}(1)}}, g_{2}^{a_{\phi^{-1}(2)}}, \cdots, g_{2}^{a_{\phi^{-1}(n)}}\right)$. It is clear that $\beta$ is an isomorphism of groups. Note $\beta(a)=\phi \cdot a$.

(iii) It is clear that $\chi \beta^{-1}=\chi^{(\nu)} \in \widehat{A^{\psi(\sigma)}}$. Indeed, for any $a=\left(g_{2}^{a_{1}}, g_{2}^{a_{2}}, \cdots, g_{2}^{a_{n}}\right) \in A^{\psi(\sigma)}$, see

$$
\begin{aligned}
\chi^{(\nu)}(a) & =\chi_{2}\left(g_{2}\right)^{a_{1}+a_{2}+\cdots+a_{\nu}} \text { and } \\
\chi \beta^{-1}(a) & =\chi_{2}\left(g_{2}\right)^{\delta_{1} a_{\phi(1)}+\delta_{2} a_{\phi(2)}+\cdots+\delta_{n} a_{\phi(n)}} \\
& =\chi_{2}\left(g_{2}\right)^{a_{1}+a_{2}+\cdots+a_{\nu}} .
\end{aligned}
$$

Thus $\chi \beta^{-1}=\chi^{(\nu)}$.

(iv) $\rho \psi^{-1} \in \widehat{\left(\mathbb{S}_{n}\right)_{\chi(\nu)}^{\psi(\sigma)}}$. In fact, it is sufficient to show that $\psi^{-1}$ is a group isomorphism from $\left(\mathbb{S}_{n}\right)_{\chi(\nu)}^{\psi(\sigma)}$ to $\left(\mathbb{S}_{n}\right)_{\chi}^{\sigma}$. For any $h \in\left(\mathbb{S}_{n}\right)_{\chi}^{\psi(\sigma)}$, we have $\psi(\sigma) h=h \psi(\sigma)$ and $h \cdot \chi^{(\nu)}=\chi^{(\nu)}$. Therefore $\sigma \psi^{-1}(h)=\psi^{-1}(h) \sigma$ and for any $a=\left(g_{2}^{a_{1}}, g_{2}^{a_{2}}, \cdots, g_{2}^{a_{n}}\right) \in A^{\sigma}$,

$$
\begin{aligned}
\left(\psi^{-1}(h) \cdot \chi\right)(a) & =\chi(\psi(h) \cdot a) \\
& =\chi\left(g_{2}^{a_{\psi^{-1}(h)(1)}}, g_{2}^{a_{\psi^{-1}(h)(2)}}, \cdots, g_{2}^{a_{\psi^{-1}(h)(n)}}\right) \\
& =\chi_{2}\left(g_{2}\right)^{\delta_{1} a_{\psi^{-1}(h)(1)}+\delta_{2} a_{\psi^{-1}(h)(2)}+\cdots+\delta_{n} a_{\psi^{-1}(h)(n)} .}
\end{aligned}
$$

Considering $\psi^{-1}(h)(i)=\phi^{-1} h \phi(i) \in W$ when $i \in W$ since $h \in\left(S_{n}\right)_{\chi^{(\nu)}}=\mathbb{S}_{\nu} \times \mathbb{S}_{\{\nu+1, \cdots, n\}}$, we have $\psi^{-1}(h) \cdot \chi=\chi$.

(v) $\beta \otimes \psi$ is a group isomorphism from $A^{\sigma} \rtimes\left(\mathbb{S}_{n}\right)_{\chi}^{\sigma}$ to $\left.A^{\psi(\sigma)} \rtimes\left(\mathbb{S}_{n}\right)_{\chi}^{\psi(\sigma)}\right)$. In fact, since $\psi(h)$. $\beta(a)=\beta(h \cdot a)$ for any $h \in\left(\mathbb{S}_{n}\right)_{\chi}^{\sigma}, a \in A^{\sigma}$ we have that $\beta \otimes \psi$ is a group homomorphism.

(vi) $\theta_{\chi^{(\nu)}, \rho \psi^{-1}}(\beta \otimes \psi)=\theta_{\chi, \rho}$. This follows from Lemma 3.3 .

Consequently, $\operatorname{RSR}\left(G, \mathcal{O}_{\sigma}, \theta_{\chi, \rho}\right) \cong \operatorname{RSR}\left(G, \mathcal{O}_{\sigma^{\prime}}, \theta_{\chi^{(\nu)}, \rho^{\prime}}\right)$ by [ZZWC, Lemma 1.8] and [ZCZ, Theorem 4], where $\rho^{\prime}=\rho \psi^{-1}$.

By lemma above, we only consider $\chi^{(\nu)}$ in $\widehat{A^{\sigma}}$ from now on. 
Lemma 3.6. Let $G=A \rtimes \mathbb{S}_{n}$ and $\sigma \in \mathbb{S}_{n}$. If $\operatorname{RSR}\left(G, \mathcal{O}_{\sigma}, \theta_{\chi^{(\nu)}, \rho}\right)$ is matched, then $\operatorname{RSR}\left(G, \mathcal{O}_{\sigma}, \theta_{\chi^{(\nu), \rho}}\right)$ is -1-type if and only if distinguished element $\xi_{\mathbf{t}, \sigma}$ of $\operatorname{RSR}\left(G, \mathcal{O}_{\sigma}\right.$, $\left.\theta_{\chi^{(\nu, \rho}, \rho}\right)$ is an integer and the order of $\sigma$ is even.

Proof. $\operatorname{RSR}\left(G, \mathcal{O}_{\sigma}, \theta_{\chi^{(\nu)}, \rho}\right)$ is -1-type if and only if so is $\operatorname{RSR}\left(\left(\mathbb{S}_{n}\right)_{\chi^{(\nu)}}, \mathcal{O}_{\sigma}, \rho\right)$ by Proposition 1.9. If $\sigma \in \mathbb{S}_{\nu}$, then $\rho=\rho^{\prime} \otimes \rho^{\prime \prime}$ with $\rho^{\prime} \in \widehat{\mathbb{S}_{\nu}^{\sigma}}$ and $\rho^{\prime \prime} \in \mathbb{S}_{\{\nu+1, \cdots, n\}}$. By Lemma 1.12, $\operatorname{RSR}\left(\left(\mathbb{S}_{n}\right)_{\chi^{(\nu)}}, \mathcal{O}_{\sigma}, \rho\right)$ is -1-type if and only if $\operatorname{RSR}\left(\mathbb{S}_{\nu}, \mathcal{O}_{\sigma}, \rho^{\prime}\right)$ is -1-type. It follows from Proposition 2.5 that $\operatorname{RSR}\left(G, \mathcal{O}_{\sigma}, \theta_{\chi^{(\nu)}, \rho}\right)$ is -1-type if and only if the distinguished element $\xi_{\mathbf{t}, \sigma}$ of $\operatorname{RSR}\left(\mathbb{S}_{\nu}, \mathcal{O}_{\sigma}, \rho^{\prime}\right)$ is an integer and the order of $\sigma$ is even.

If $\sigma \in \mathbb{S}_{\{\nu+1, \cdots, n\}}$, then $\rho=\rho^{\prime} \otimes \rho^{\prime \prime}$ with $\rho^{\prime} \in \widehat{\mathbb{S}_{\nu}}$ and $\rho^{\prime \prime} \in \mathbb{S}_{\{\nu+1, \cdots, n\}}^{\sigma}$. By Lemma 1.12, $\operatorname{RSR}\left(\left(\mathbb{S}_{n}\right)_{\chi^{(\nu)}}, \mathcal{O}_{\sigma}, \rho\right)$ is -1-type if and only if $\operatorname{RSR}\left(\mathbb{S}_{\{\nu+1, \cdots, n\}}, \mathcal{O}_{\sigma}, \rho^{\prime \prime}\right)$ is -1-type. It follows from Proposition 2.5 that $\operatorname{RSR}\left(G, \mathcal{O}_{\sigma}, \theta_{\chi^{(\nu)}, \rho}\right)$ is -1-type if and only if the distinguished element $\xi_{\mathbf{t}, \sigma}$ of $\operatorname{RSR}\left(\mathbb{S}_{\{\nu+1, \cdots, n\}}, \mathcal{O}_{\sigma}, \rho^{\prime \prime}\right)$ is an integer and the order of $\sigma$ is even.

The proof of Theorem 2, We only show this under the case of $\sigma \in \mathbb{S}_{\nu}$ since the other is similar. By Proposition 2.8 and Lemma 3.6, $\mathfrak{B}\left(\mathcal{O}_{\sigma}^{G}, \theta_{\chi^{(\nu), \rho}}\right)$ is -1-type under the cases in Theorem 2, See

$$
\begin{aligned}
\operatorname{RSR}\left(\mathbb{S}_{\nu}, \mathcal{O}_{\sigma}, \rho^{\prime}\right) & \left.\leq \operatorname{RSR}\left(\left(\mathbb{S}_{n}\right)_{\chi^{(\nu)}}, \mathcal{O}_{\sigma}, \rho^{\prime} \otimes \rho^{\prime \prime}\right) \quad \text { by Lemma } 1.11(i i i)\right) \\
& \leq \operatorname{RSR}\left(G, \mathcal{O}_{\sigma}, \theta_{\chi^{(\nu)}, \rho}\right) \quad(\text { by Proposition 1.9(iii)), }
\end{aligned}
$$

which implies $\operatorname{RSR}\left(\mathbb{S}_{\nu}, \mathcal{O}_{\sigma}, \rho^{\prime}\right) \leq \operatorname{RSR}\left(G, \mathcal{O}_{\sigma}, \theta_{\chi^{(\nu)}, \rho}\right)$. By Proposition[1.6(iii), $\operatorname{dim} \mathfrak{B}\left(\mathbb{S}_{\nu}, \mathcal{O}_{\sigma}\right.$, $\mu)<\infty$. Applying [AFZ, Theorem 1] we complete the proof.

Proposition 3.7. Let $G=A \rtimes \mathbb{S}_{n}$ with $\sigma \in \mathbb{S}_{n}$. Then $\left(\mathbb{S}_{n}\right)_{\chi^{(\nu)}}^{\sigma}=\left(\mathbb{S}_{n}\right)^{\sigma}$ if and only if $Y_{j} \subseteq\{1,2, \cdots, \nu\}$ or $Y_{j} \subseteq\{\nu+1, \nu+2, \cdots, n\}$ for $1 \leq j \leq n$, where $Y_{j}$ is the same as in the begin of Section Q

Proof. If $Y_{j} \subseteq\{1,2, \cdots, \nu\}$, then $A_{l, j}, B_{h, j} \in \mathbb{S}_{\{1,2, \cdots, \nu\}}$ for $1 \leq l \leq \lambda_{j}$ and $1 \leq h \leq \lambda_{j}-$ 1 , where $A_{l, j}, B_{h, j}$ are the same as in the begin of Section 2. If $Y_{j} \subseteq\{\nu+1, \nu+2, \cdots, n\}$, then $A_{l, j}, B_{h, j} \in \mathbb{S}_{\{\nu+1, \nu+2, \cdots, n\}}$ for $1 \leq l \leq \lambda_{j}$ and $1 \leq h \leq \lambda_{j}-1$. Consequently, $\left(\mathbb{S}_{n}\right)_{\chi(\nu)}^{\sigma}=\left(\mathbb{S}_{n}\right)^{\sigma}$

Conversely, assume $\left(\mathbb{S}_{n}\right)_{\chi(\nu)}^{\sigma}=\left(\mathbb{S}_{n}\right)^{\sigma}$. If there exists $1 \leq j \leq n$ such that $Y_{j} \nsubseteq$ $\{1,2, \cdots, \nu\}$ and $Y_{j} \nsubseteq\{\nu+1, \nu+2, \cdots, n\}$, then there exist $a, b \in Y_{j}$ with $a \in\{1,2, \cdots, \nu\}$ and $b \in\{\nu+1, \nu+2, \cdots, n\}$. Note $Y_{j}=\left\{y_{s} \mid s=r_{j}+1, \cdots, r_{j+1}\right\}$.

If there exists $l$ such that $a, b \in\left\{y_{r_{j}+(l-1) j+1}, \quad y_{r_{j}+(l-1) j+2}, \cdots, \quad y_{r_{j}+l j}\right\}$, then $A_{l, j} \notin\left(\mathbb{S}_{n}\right)_{\chi(\nu)}^{\sigma}$, a contradiction. Thus there exist $l \neq l^{\prime}$ such that $a \in A_{l, j}$ and $b \in$ $A_{l^{\prime}, j}$. Let $a=y_{r_{j}+(l-1) j+s}$ and $b=y_{r_{j}+\left(l^{\prime}-1\right) j+s^{\prime}}$. Considering $B_{h, j} \in\left(\mathbb{S}_{n}\right)_{\chi^{(\nu)}}^{\sigma}$, we have $y_{r_{j}+\left(l^{\prime}-1\right) j+s} \in\{1,2, \cdots, \nu\}$ which is a contradiction.

3.2. Central quantum linear space. A central quantum linear space is a finite dimensional Nichols algebra, which was introduced in [ZZWC, Def. 2.12]. $\operatorname{RSR}(G, r, \vec{\rho}, u)$ 
is said to be a central quantum linear type if it is quantum symmetric and of the nonessentially infinite type with $C \subseteq Z(G)$ for any $C \in \mathcal{K}_{r}(G)$. In this case, $\mathfrak{B}(G, r, \vec{\rho}, u)$ is called a central quantum linear space over $G$.

We give the other main result.

Theorem 3. $\mathfrak{B}(G, r, \vec{\rho}, u)$ is a central quantum linear space over classical Weyl group $G$ if and only if $C=\left\{\left(g_{2}, \cdots, g_{2}\right)\right\} \subseteq G, r=r_{C} C, \rho_{C}^{(i)}=\theta_{\chi_{C}^{(i)}, \mu_{C}^{(i)}}:=\left(\chi_{C}^{(i)} \otimes \mu_{C}^{(i)}\right) \uparrow_{\substack{G^{u(C)} \\ G_{C}^{(i)}}}^{(i)}$ $\widehat{G^{u(C)}}$ with $\chi_{C}^{(i)} \in\left\{\chi_{2}^{\delta_{1}^{(i)}} \otimes \chi_{2}^{\delta_{2}^{(i)}} \otimes \cdots \otimes \chi_{2}^{\delta_{n}^{(i)}} \mid \delta_{1}^{(i)}+\delta_{2}^{(i)}+\cdots+\delta_{n}^{(i)}\right.$ is odd $\}$ for any $i \in I_{C}(r, u)$.

Proof. It is clear $\theta_{\chi_{C}^{(i)}, \rho_{C}^{(i)}}\left(\left(g_{2}, \cdots, g_{2}\right)\right)=\chi_{2}\left(g_{2}\right)^{\delta_{1}^{(i)}+\delta_{2}^{(i)}+\cdots+\delta_{n}^{(i)}}$ id as in the proof of Lemma 2.1 (ii). Applying [ZZWC, Remark 3.16], we complete the proof.

In other words we have

Remark 3.8. Let $G=A \rtimes \mathbb{S}_{n}$. Assume that $a=\left(g_{2}, g_{2}, \cdots, g_{2}\right) \in G$ and $M=$ $M\left(\mathcal{O}_{a}, \rho^{(1)}\right) \oplus M\left(\mathcal{O}_{a}, \rho^{(2)}\right) \oplus \cdots \oplus M\left(\mathcal{O}_{a}, \rho^{(m)}\right)$ is a YD module over $k G$ with $\rho^{(i)}=$ $\theta_{\chi^{\left(\nu_{i}\right)}, \mu^{(i)}}:=\left(\chi^{\left(\nu_{i}\right)} \otimes \mu^{(i)}\right) \uparrow_{G_{\chi\left(\nu_{i}\right)}^{a}}^{a} \in \widehat{G^{a}}$ and odd $\nu_{i}$ for $i=1,2, \cdots, m$. Then $\mathfrak{B}(M)$ is finite dimensional.

3.3. Reducible Yetter-Drinfeld modules. $\mathcal{O}_{\sigma}$ and $\mathcal{O}_{\tau}$ are said to be square-commutative if stst $=$ tsts for any $s \in \mathcal{O}_{\sigma}, t \in \mathcal{O}_{\tau}$. Obviously, $\mathcal{O}_{\sigma}$ and $\mathcal{O}_{\tau}$ are square-commutative if and only if sts $\in G^{t}$ for any $s \in \mathcal{O}_{\sigma}, t \in \mathcal{O}_{\tau}$ if and only if $s \tau s \in G^{\tau}$ for any $s \in \mathcal{O}_{\sigma}$.

Lemma 3.9. Let $G=A \rtimes D$. Let $(a, \sigma),(b, \tau) \in G$ with $a, b \in A, \sigma, \tau \in D$. If $\mathcal{O}_{(a, \sigma)}^{G}$ and $\mathcal{O}_{(b, \tau)}^{G}$ are square-commutative then $\mathcal{O}_{\sigma}^{D}$ and $\mathcal{O}_{\tau}^{D}$ are square-commutative.

Proof. It is clear that $(a, \sigma)^{-1}=\left(\sigma^{-1} \cdot a^{-1}, \sigma^{-1}\right)$ and

$$
(b, \tau)(a, \sigma)(b, \tau)^{-1}=\left(b(\tau \cdot a)\left(\tau \sigma \tau^{-1} \cdot b^{-1}\right), \tau \sigma \tau^{-1}\right) .
$$

For any $x \in \mathcal{O}_{\sigma}^{D}$ and $y \in \mathcal{O}_{\tau}^{D}$, by (3.2), there exist $c, d \in A$ such that $(c, x) \in \mathcal{O}_{(a, \sigma)}^{G}$ and $(d, y) \in \mathcal{O}_{(b, \tau)}^{G}$. Since $(c, x)(d, y)(c, x)(d, y)=(d, y)(c, x)(d, y)(c, x)$, we have $x y x y=y x y x$, i.e. $\mathcal{O}_{\sigma}^{D}$ and $\mathcal{O}_{\tau}^{D}$ are square-commutative.

Lemma 3.10. Let $1 \neq \sigma, 1 \neq \tau \in \mathbb{S}_{n}$ with $G=\mathbb{S}_{n}$ and $n>2$. If $\mathcal{O}_{\sigma}$ and $\mathcal{O}_{\tau}$ are square-commutative, then one of the following conditions holds.

(i) $n=3, \mathcal{O}_{\sigma}=\mathcal{O}_{\tau}=\mathcal{O}_{(123)}$ or $\mathcal{O}_{\sigma}=\mathcal{O}_{(12)}$ and $\mathcal{O}_{\tau}=\mathcal{O}_{(123)}$.

(ii) $n=4, \mathcal{O}_{\sigma}=\mathcal{O}_{\tau}=\mathcal{O}_{(12)(34)}$ or $\mathcal{O}_{\sigma}=\mathcal{O}_{(12)(34)}$ and $\mathcal{O}_{\tau}=\mathcal{O}_{(1234)}$ or $\mathcal{O}_{\sigma}=\mathcal{O}_{(12)}$ and $\mathcal{O}_{\tau}=\mathcal{O}_{(12)(34)}$.

(iii) $n=2 k$ with $k>2, \mathcal{O}_{\sigma}=\mathcal{O}_{(12)}$ and $\mathcal{O}_{\tau}=\mathcal{O}_{(12)(34) \cdots(n-1 n)}$.

Proof. We show this by following several steps. Assume that $1^{\lambda_{1}} 2^{\lambda_{2}} \cdots n^{\lambda_{n}}$ and $1^{\lambda_{1}^{\prime}} 2^{\lambda_{2}^{\prime}} \cdots n^{\lambda_{n}^{\prime}}$ are the types of $\sigma$ and $\tau$, respectively; $\mathcal{O}_{\sigma}$ and $\mathcal{O}_{\tau}$ are square-commutative.

(i) Let $n=3$. Obviously, $\mathcal{O}_{(12)}$ and $\mathcal{O}_{(12)}$ are not square-commutative. Then $\mathcal{O}_{\sigma}=$ $\mathcal{O}_{\tau}=\mathcal{O}_{(123)}$ or $\mathcal{O}_{\sigma}=\mathcal{O}_{(12)}$ and $\mathcal{O}_{\tau}=\mathcal{O}_{(123)}$. 
(ii) Let $n=4$. The types of $\sigma$ and $\tau$ are $2^{2} ; 4^{1} ; 1^{1} 3^{1} ; 1^{2} 2^{1}$, respectively.

(a). $\mathcal{O}_{(12)(34)}$ and $\mathcal{O}_{(123)}$ are not square-commutative since $(12)(34)(123)(12)(34)=$ (214), which implies $(12)(34)(123)(12)(34) \notin G^{(123)}$.

(b). $\mathcal{O}_{(1234)}$ and $\mathcal{O}_{(1234)}$ are not square-commutative since $\quad((1234)(4231))^{2} \quad$ and $((4231)(1234))^{2}$ maps 1 to 1 and 2 , respectively.

(c). $\mathcal{O}_{(1234)}$ and $\mathcal{O}_{(123)}$ are not square-commutative since $(1234)(123)(1234)$ maps 1 to 4.

(d). $\mathcal{O}_{(1234)}$ and $\mathcal{O}_{(12)}$ are not square-commutative since $(1234)(12)(1234)$ maps 2 to 4.

(e). $\mathcal{O}_{(123)}$ and $\mathcal{O}_{(123)}$ are not square-commutative since $(134)(123)(134)$ maps 2 to 4.

(f). $\mathcal{O}_{(123)}$ and $\mathcal{O}_{(12)}$ are not square-commutative since $(14)(123)(14)=(423)$.

(g). $\mathcal{O}_{(12)}$ and $\mathcal{O}_{(12)}$ are not square-commutative since $(14)(12)(14)=(42)$.

(iii) If $\sigma=(12 \cdots r)$ with $n>4$, then $r=n$ or $\mathcal{O}_{\sigma}=\mathcal{O}_{(12)}$ and $\mathcal{O}_{\tau}=\mathcal{O}_{(12)(34) \cdots(n-1 n)}$ with $n=2 k>2$. In fact, obviously, $G^{\sigma}$ is a cycle group generated by $(12 \cdots r)$.

(a). If $\lambda_{3}^{\prime} \neq 0$ and $n-r>1$, set $t=(\cdots, r, r+1, r+2 \cdots) t_{1}$, an independent decomposition of $t \in \mathcal{O}_{\tau}$. See $t \sigma t(r)=r+2$, which implies $t \sigma t \notin G^{\sigma}$.

(b). If $\lambda_{3}^{\prime} \neq 0$ and $n-r=1$. Set $t=(1, r, r+1) t_{1}$, an independent decomposition of $t \in \mathcal{O}_{\tau}$. See $t \sigma t(r+1)=t(2)<r+1$ since $r \neq 2$, which implies $t \sigma t \notin G^{\sigma}$.

(c). If there exists $j>3$ such that $\lambda_{j}^{\prime} \neq 0$ with $n>r>2$, set $t=(\cdots, r-2, r-$ $1, r, r+1, \cdots) t_{1}$, an independent decomposition of $t \in \mathcal{O}_{\tau}$. See $t \sigma t(r-2)=r+1$, which implies $t \sigma t \notin G^{\sigma}$.

(d). If there exists $j>3$ such that $\lambda_{j}^{\prime} \neq 0$ with $r=2$, set $t=(123 \cdots) t_{1}$, an independent decomposition of $t \in \mathcal{O}_{\tau}$. See $t \sigma t(2)=4$, which implies $t \sigma t \notin G^{\sigma}$.

(e). If the type of $\tau$ is $1^{\lambda_{1}^{\prime}} 2^{1}$ with $n>r$, set $t=(r r+1) \in \mathcal{O}_{\tau}$. See $t \sigma t=(1 \cdots r r+1) \notin$ $G^{\sigma}$.

(f). If the type of $\tau$ is $1^{\lambda_{1}^{\prime}} 2^{\lambda_{2}^{\prime}}$ with $n>r>2$ and $\lambda_{2}^{\prime}>1$, set $t=(r r+1)(12) t_{1}$, an independent decomposition of $t \in \mathcal{O}_{\tau}$. See $t \sigma t=(21 \cdots r+1) \notin G^{\sigma}$.

(g). If the type of $\tau$ is $1^{\lambda_{1}^{\prime}} 2^{\lambda_{2}^{\prime}}$ with $r=2, \lambda_{2}^{\prime} \geq 1$ and $\lambda_{1}^{\prime} \neq 0$, set $t=(1)(23) t_{1}$, an independent decomposition of $t \in \mathcal{O}_{\tau}$. See $t \sigma t=(13) \notin G^{\sigma}$.

From now on assume that both $\sigma$ and $\tau$ are not cycles.

(iv) If $n>4$ and $\sigma=(1,2, \cdots, n)$ is a cycle, then it is a contradiction.

(a). If $\lambda_{2}^{\prime} \neq 0$, set $t=(1, n)(2,3, \cdots) t_{1}$, an independent decomposition of $t \in \mathcal{O}_{\tau}$. See $t \sigma t \sigma(1)=t(4) \neq 1$ and $\sigma t \sigma t(1)=1$.

(b). If $\lambda_{3}^{\prime} \neq 0$, set $t=(123) t_{1}$, an independent decomposition of $t \in \mathcal{O}_{\tau}$. See $t \sigma t \sigma(1)=$ $t(4)$ and $\sigma t \sigma t(1)=2$, which implies that $t \sigma t \notin G^{\sigma}$ since $t(4)>3$.

(c). If $\lambda_{4}^{\prime} \neq 0$, set $t=(1234) t_{1}$, an independent decomposition of $t \in \mathcal{O}_{\tau}$. See $t \sigma t \sigma(1)=1$ and $\sigma t \sigma t(1)=5$, which implies $t \sigma t \notin G^{\sigma}$.

(d). If $n>5$ and there exists $j>4$ such that $\lambda_{j}^{\prime} \neq 0$, set $t=(12346 \cdots) t_{1}$, an independent decomposition of $t \in \mathcal{O}_{\tau}$. See $\sigma t \sigma t(1)=5$ and $t \sigma t \sigma(1)=6$, which implies that $t \sigma t \notin G^{\sigma}$. 
(e). If $n=5$ and there exists $j>4$ such that $\lambda_{j}^{\prime} \neq 0$, set $t=(13254) \in \mathcal{O}_{\tau}$. See $\sigma t \sigma t(1)=2$ and $t \sigma t \sigma(1)=3$, which implies that $t \sigma t \notin G^{\sigma}$.

(v) If $n>4$ and there exists $r>1$ such that $\lambda_{r}>1$, then $n=\lambda_{r} r$. Let $\sigma=$ $(1,2, \cdots, r)(r+1, \cdots, 2 r) \cdots\left(\left(\lambda_{r}-1\right) r+1, \cdots, \lambda_{r} r\right) \sigma_{1}$, an independent decomposition of $\sigma$. Assume $n>\lambda_{r} r$.

(a) If $r>2$ and $\lambda_{j}^{\prime}=0$ for any $j>2$, set $t=(1, n)(2,3) t_{1}$, an independent decomposition of $t \in \mathcal{O}_{\tau}$. See $t \sigma t=\left(n, 3, a_{3}, \cdots, a_{r}\right) \cdots$, which implies that $t \sigma t \notin G^{\sigma}$.

(b). If $r=2$ and $\lambda_{j}^{\prime}=0$ for any $j>2$, set $t=(1, n)(2,3) t_{1}$, an independent decomposition of $t \in \mathcal{O}_{\tau}$. See $t \sigma t=(n, 3)(2, t(4)) \cdots$, which implies that $t \sigma t \notin G^{\sigma}$ since $t(4)>2$.

(c). If there exists $j>2$ such that $\lambda_{j}^{\prime} \neq 0$, set $t=\left(1, a_{1}, \cdots, a_{p}, \lambda_{r} r, \lambda_{r} r+1\right)(2,3, \cdots) t_{1}$, an independent decomposition of $t \in \mathcal{O}_{\tau}$. See $t \sigma t\left(\lambda_{r} r+1\right)=t \sigma(1)=3$, which implies that $t \sigma t \notin G^{\sigma}$.

(vi) If $n>4$ and $\lambda_{r} \leq 1$ for any $r>1$, then this is a contradiction. Assume that there exist $r$ and $r^{\prime}$ such that $\lambda_{r^{\prime}} \neq 0$ and $\lambda_{r} \neq 0$ with $2 \leq r^{\prime}<r$. Let $\sigma=(12 \cdots r)(r+1 \cdots r+$ $\left.r^{\prime}\right) \sigma_{1}$ be an independent decomposition of $\sigma$.

(a). If $\lambda_{i}^{\prime}=0$ for any $i>2$, set $t=(1 n)(23) t_{1}$, an independent decomposition of $t \in \mathcal{O}_{\tau}$. See $t \sigma t=(n 3 \cdots) \cdots$, which implies that $t \sigma t \notin G^{\sigma}$.

(b). If $r>3$ and there exists $j>2$ such that $\lambda_{j}^{\prime} \neq 0$, set $t=\left(1, a_{1}, \cdots, a_{p}, r, r+\right.$ 1) $(2,3, \cdots) t_{1}$, an independent decomposition of $t \in \mathcal{O}_{\tau}$. See $\operatorname{t} \sigma t(r+1)=3$, which implies that $t \sigma t \notin G^{\sigma}$.

(c). If $r=3$ and there exists $j>2$ such that $\lambda_{j}^{\prime} \neq 0$, set $t=(123 \cdots)(34 \cdots) t_{1}$, an independent decomposition of $t \in \mathcal{O}_{\tau}$. See $t \sigma t(1)=4$, which implies that $t \sigma t \notin G^{\sigma}$.

(vii) If $n>4$ and the types of $\sigma$ and $\tau$ are $r^{\lambda_{r}}$, then it is a contradiction. Let $\sigma=(12 \cdots r)(r+1 \cdots 2 r) \cdots$. Set $t=(r+1,2, \cdots, r)(1, r+2, \cdots, 2 r) t_{1}$, an inde-

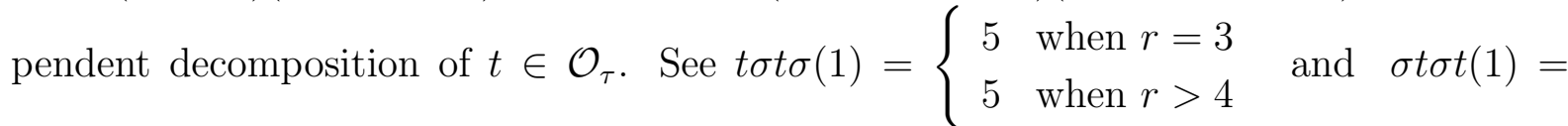
$\left\{\begin{array}{ll}2 & \text { when } r=3 \\ r+5 & \text { when } r>4\end{array}\right.$. When $r=4$, set $t=(4231) t_{1}$, an independent decomposition of $t \in \mathcal{O}_{\tau} . \sigma t \sigma t(1)=1$ and $t \sigma t \sigma(1)=2$. When $r=2$, set $t=(14)(25)(36) t_{1}$, an independent decomposition of $t \in \mathcal{O}_{\tau}$. See $\sigma t \sigma t(1)=5$ and $t \sigma t \sigma(1)=3$. Then $\sigma t \sigma t \neq t \sigma t \sigma$.

In fact, $\mathcal{O}_{\sigma}$ and $\mathcal{O}_{\tau}$ in cases of Lemma 3.10 (i) (ii) are square- commutative .

Lemma 3.11. Let $G=A \rtimes \mathbb{S}_{n}$ with $A \subseteq\left(C_{2}\right)^{n}$. Then

(i) $n=3, \mathcal{O}_{(a,(123))}$ and $\mathcal{O}_{(b,(123))}$ are not square-commutative.

(ii) $n=3, \mathcal{O}_{(a,(12))}$ and $\mathcal{O}_{(b,(123))}$ are not square-commutative.

(iii) $n=4, \mathcal{O}_{(a,(12)(34))}$ and $\mathcal{O}_{(b,(1234))}$ are not square-commutative.

(iv) $n=2 k$ with $k>1, \mathcal{O}_{(a,(12))}$ and $\mathcal{O}_{(b,(12)(34))}$ are not square-commutative. 
$(\mathrm{v})$ If $n=4$, then $\mathcal{O}_{(a,(12)(34))}$ and $\mathcal{O}_{(b,(12)(34))}$ are square-commutative if and only if the signs of $(a,(12)(34))$ and $(b,(12)(34))$.

Proof. For any $(d, \mu) \in G=A \rtimes \mathbb{S}_{n}$, let $\left(c, \mu \sigma \mu^{-1}\right):=(d, \mu)(a, \sigma)(d, \mu)^{-1}$, i.e. $c=$ $d(\mu \cdot a)\left(\mu \sigma \mu^{-1} \cdot d\right)$. It is clear that $\left(\left(c, \mu \sigma \mu^{-1}\right)(b, \tau)\right)^{2}=\left((b, \tau)\left(c, \mu \sigma \mu^{-1}\right)\right)^{2}$ if and only if

$$
\begin{aligned}
& d(\mu \cdot a)\left(\mu \sigma \mu^{-1} \cdot d\right)\left(\mu \sigma \mu^{-1} \cdot b\right)\left(\mu \sigma \mu^{-1} \tau \cdot d\right) \\
& \left(\mu \sigma \mu^{-1} \tau \mu \cdot a\right)\left(\mu \sigma \mu^{-1} \tau \mu \sigma \mu^{-1} \cdot d\right)\left(\mu \sigma \mu^{-1} \tau \mu \sigma \mu^{-1} \cdot b\right) \\
= & b(\tau \cdot d)(\tau \mu \cdot a)\left(\tau \mu \sigma \mu^{-1} \cdot d\right)\left(\tau \mu \sigma \mu^{-1} \cdot b\right)\left(\tau \mu \sigma \mu^{-1} \tau \cdot d\right) \\
& \left(\tau \mu \sigma \mu^{-1} \tau \mu \cdot a\right)\left(\tau \mu \sigma \mu^{-1} \tau \mu \sigma \mu^{-1} \cdot d\right),
\end{aligned}
$$

which is equivalent to

$$
\begin{aligned}
& d\left(\mu \sigma \mu^{-1} \cdot d\right)\left(\mu \sigma \mu^{-1} \tau \cdot d\right)\left(\mu \sigma \mu^{-1} \tau \mu \sigma \mu^{-1} \cdot d\right)(\tau \cdot d)\left(\tau \mu \sigma \mu^{-1} \cdot d\right)\left(\tau \mu \sigma \mu^{-1} \tau \cdot d\right) \\
& \left(\tau \mu \sigma \mu^{-1} \tau \mu \sigma \mu^{-1} \cdot d\right)=h
\end{aligned}
$$

with $h:=(\mu \cdot a)\left(\mu \sigma \mu^{-1} \cdot b\right)\left(\mu \sigma \mu^{-1} \tau \mu \cdot a\right)\left(\mu \sigma \mu^{-1} \tau \mu \sigma \mu^{-1} \cdot b\right) b(\tau \mu \cdot a)\left(\tau \mu \sigma \mu^{-1} \cdot b\right)\left(\tau \mu \sigma \mu^{-1} \tau \mu \cdot a\right)$.

We only need to show that there exists $(d, \mu) \in G$ such that (3.4) does not hold in the four cases above, respectively. Let $d=\left(g_{2}^{d_{1}}, g_{2}^{d_{2}}, \cdots, g_{2}^{d_{n}}\right)$ for any $d \in A$.

(i) Let $\sigma=(123)=\tau=\mu$ and $n=3$. (3.4) becomes $d(\sigma \cdot d)=h$, which implies $d_{1}+d_{3} \equiv h_{1}(\bmod 2)$. This is a contradiction since $d$ has not this restriction.

(ii) Let $\tau=(123), \sigma=(12)=\mu$ and $n=3$. (3.4) becomes $\left(\tau^{-1} \cdot d\right)((32) \cdot d)(\tau \cdot d)((13)$. $d)=h$, which implies $d_{1}+d_{2} \equiv h_{1}(\bmod 2)$. This is a contradiction since $d$ has not this restriction.

(iii) Let $\sigma=(12)(34), \tau=(1234), \mu=(123)$ and $n=4$. (3.4) becomes ((13) . $d)((4321) \cdot d)((1234) \cdot d)((24) \cdot d)=h$, which implies $d_{1}+d_{2}+d_{3}+d_{4} \equiv h_{1}(\bmod 2)$. This is a contradiction since $d$ has not this restriction.

(iv) Let $\sigma=(12), \lambda=(56)(78) \cdots(n-1 n), \tau=(12)(34) \lambda, \mu=(123)$. (3.4) becomes

$$
\begin{aligned}
& d((23) \cdot d)((1342) \lambda \cdot d)((13)(24) \lambda \cdot d)((12)(34) \lambda \cdot d)((1243) \lambda \cdot d)((14) \cdot d) \\
& ((14)(23) \cdot d)=h,
\end{aligned}
$$

which implies $0 \equiv h_{i}(\bmod 2)$ for $i=1, \cdots, n$. By simple computation, we have $(\mu$. $a)((1342) \mu \cdot a)((12)(34) \mu \cdot a)((14) \mu \cdot a)((23) \cdot b)((13)(24) \cdot b) b((1243) \cdot b)=1$, which implies $a_{3}+a_{4} \equiv 0(\bmod 2)$. If $(a, \sigma)$ is a negative cycle, we construct a negative cycle $\left(a^{\prime}, \sigma\right)$ such that $a_{4}^{\prime} \equiv a_{3}^{\prime}$ does not hold as follows: Let $a_{i}^{\prime}=0$ when $i \neq 3,4$, and $a_{4}^{\prime}=1$, $a_{3}^{\prime}=0$. If $(a, \sigma)$ is a positive cycle, we construct a positive cycle $\left(a^{\prime}, \sigma\right)$ such that $a_{4}^{\prime} \equiv a_{3}^{\prime}$ does not hold as follows: Let $a_{i}^{\prime}=0$ when $i \neq 1,3,4$, and $a_{4}^{\prime}=1, a_{3}^{\prime}=0, a_{1}^{\prime}=1$. Since $\mathcal{O}_{(a, \sigma)}=\mathcal{O}_{\left(a^{\prime}, \sigma\right)}$, we obtain a contradiction.

(v) It is clear that $\mathcal{O}_{(12)(34)}^{\mathbb{S}_{4}}$ and $\mathcal{O}_{(12)(34)}^{\mathbb{S}_{4}}$ are commutative. (3.4) becomes $1=h$. That is, $(\mu \cdot a)\left(\mu \sigma \mu^{-1} \sigma \mu \cdot a\right)(\sigma \mu \cdot a)\left(\mu \sigma \mu^{-1} \mu \cdot a\right)\left(\mu \sigma \mu^{-1} \cdot b\right)(\sigma \cdot b) b\left(\sigma \mu \sigma \mu^{-1} \cdot b\right)=1$. It is clear that $\sigma(i), \mu \sigma \mu^{-1}(i),(1)(i)$ and $\mu \sigma \mu^{-1} \sigma(i)$ are different each other for any fixed $i$ with 
$1 \leq i \leq 4$ when $\mu \sigma \mu^{-1} \neq \sigma$. Therefore, (3.4) holds for any $(d, \mu) \in A \rtimes \mathbb{S}_{n}$ if and only if $a_{1}+a_{2}+a_{3}+a_{4} \equiv b_{1}+b_{2}+b_{3}+b_{4}(\bmod 2)$.

We give the third main result:

Theorem 4. Let $G=A \rtimes \mathbb{S}_{n}$ with $A \subseteq\left(C_{2}\right)^{n}$ and $n>2$. Assume that there exist different two pairs $\left.\left(u\left(C_{1}\right), i_{1}\right)\right)$ and $\left.\left(u\left(C_{2}\right), i_{2}\right)\right)$ with $C_{1}, C_{2} \in \mathcal{K}_{r}(G), i_{1} \in I_{C_{1}}(r, u)$ and $i_{2} \in I_{C_{2}}(r, u)$ such that $u\left(C_{1}\right), u\left(C_{2}\right) \notin A$. If $\operatorname{dim} \mathfrak{B}(G, r, \vec{\rho}, u)<\infty$, then the following conditions hold:

(i) $n=4$.

(ii) The type of $u(C)$ is $2^{2}$ for any $u(C) \notin A$ and $C \in \mathcal{K}_{r}(G)$.

(iii) The signs of $u(C)$ and $u\left(C^{\prime}\right)$ are the same for any $u(C), u\left(C^{\prime}\right) \notin A$ and $C, C^{\prime} \in$ $\mathcal{K}_{r}(G)$.

Proof. If $\operatorname{dim} \mathfrak{B}(G, r, \vec{\rho}, u)<\infty$, then by [HS, Theorem 8.6], $\mathcal{O}_{u\left(C_{1}\right)}^{G}$ and $\mathcal{O}_{u\left(C_{2}\right)}^{G}$ are square-commutative. Let $(a, \sigma):=u\left(C_{1}\right)$ and $(b, \tau):=u\left(C_{2}\right)$. It follows from Lemma 3.9 that $\mathcal{O}_{\sigma}^{\mathbb{S}_{n}}$ and $\mathcal{O}_{\tau}^{\mathbb{S}_{n}}$ are square-commutative. Considering Lemma 3.10, we have one of the following conditions are satisfied

(i) $n=3, \mathcal{O}_{\sigma}^{\mathbb{S}_{n}}=\mathcal{O}_{\tau}^{\mathbb{S}_{n}}=\mathcal{O}_{(123)}^{\mathbb{S}_{n}}$ or $\mathcal{O}_{\tau}^{\mathbb{S}_{n}}=\mathcal{O}_{(12)}$ and $\mathcal{O}_{\sigma}^{\mathbb{S}_{n}}=\mathcal{O}_{(123)}^{\mathbb{S}_{n}}$.

(ii) $n=4, \mathcal{O}_{\sigma}^{\mathbb{S}_{n}}=\mathcal{O}_{\tau}^{\mathbb{S}_{n}}=\mathcal{O}_{(12)(34)}^{\mathbb{S}_{n}}$ or $\mathcal{O}_{\tau}^{\mathbb{S}_{n}}=\mathcal{O}_{(1234)}$ and $\mathcal{O}_{\sigma}^{\mathbb{S}_{n}}=\mathcal{O}_{(12)(34)}^{\mathbb{S}_{n}}$. Considering Lemma 3.11, we complete the proof.

In other words, we have

Remark 3.12. Let $G=A \rtimes \mathbb{S}_{n}$ with $A \subseteq\left(C_{2}\right)^{n}$ and $n>2$. Let $M=M\left(\mathcal{O}_{\sigma_{1}}, \rho^{(1)}\right) \oplus$ $M\left(\mathcal{O}_{\sigma_{2}}, \rho^{(2)}\right) \cdots \oplus M\left(\mathcal{O}_{\sigma_{m}}, \rho^{(m)}\right)$ be a reducible YD module over $k G$. Assume that there exist $i \neq j$ such that $\sigma_{i}, \sigma_{j} \notin A$. If $\operatorname{dim} \mathfrak{B}(M)<\infty$, then $n=4$, the type of $\sigma_{p}$ is $2^{2}$ and the sign of $\sigma_{p}$ is stable for any $1 \leq p \leq m$ with $\sigma_{p} \notin A$.

\section{REFERENCES}

[AFZ] Nicols Andruskiewitsch, Fernando Fantino, Shouchuan Zhang, On pointed Hopf algebras associated with the symmetric groups, Manuscripta Math., 128(2009) 3, 359-371. Also in arXiv:0807.2406.

[AS98] N. Andruskiewitsch and H. J. Schneider, Lifting of quantum linear spaces and pointed Hopf algebras of order $p^{3}$, J. Alg. 209 (1998), 645-691.

[AS00] N. Andruskiewitsch and H. J. Schneider, Finite quantum groups and Cartan matrices, Adv. Math. 154 (2000), 1-45.

[AZ07] N. Andruskiewitsch and Shouchuan Zhang, On pointed Hopf algebras associated to some conjugacy classes in $\mathbb{S}_{n}$, Proc. Amer. Math. Soc. 135 (2007), 2723-2731.

[Ca72] R. W. Carter, Conjugacy classes in the Weyl group, Compositio Mathematica, 25(1972)1, 1-59.

[Gr00] M. Graña, On Nichols algebras of low dimension, Contemp. Math. 267 (2000),111-134.

[He06] I. Heckenberger, Classification of arithmetic root systems, preprint, arXiv:math.QA/0605795.

[HS] I. Heckenberger and H.-J. Schneider, Root systems and Weyl groupoids for Nichols algebras, preprint arXiv:0807.0691.

[Mo93] S. Montgomery, Hopf algebras and their actions on rings. CBMS Number 82, Published by AMS, 1993. 
[OZ04] F. Van Oystaeyen and P. Zhang, Quiver Hopf algebras, J. Alg. 280 (2004), 577-589.

[Ra] D. E. Radford, The structure of Hopf algebras with a projection, J. Alg. 92 (1985), 322-347.

[Sa01] Bruce E. Sagan, The Symmetric Group: Representations, Combinatorial Algorithms, and Symmetric Functions, Second edition, Graduate Texts in Mathematics 203, Springer-Verlag, 2001.

[Se] Jean-Pierre Serre, Linear representations of finite groups, Springer-Verlag, New York 1977.

[Su78] Michio Suzuki, Group Theory I, Springer-Verlag, New York, 1978.

[ZZC] Shouchuan Zhang, Y-Z Zhang and H. X. Chen, Classification of PM Quiver Hopf Algebras, J. Alg. and Its Appl. 6 (2007)(6), 919-950. Also see in math.QA/0410150.

[ZCZ] Shouchuan Zhang, H. X. Chen and Y-Z Zhang, Classification of Quiver Hopf Algebras and Pointed Hopf Algebras of Nichols Type, preprint arXiv:0802.3488.

[ZWW] Shouchuan Zhang, Min Wu and Hengtai Wang, Classification of Ramification Systems for Symmetric Groups, Acta Math. Sinica, 51 (2008) 2, 253-264. Also in math.QA/0612508.

[ZZWC] Shouchuan Zhang, Y-Z Zhang, Peng Wang, Jing Cheng, On Pointed Hopf Algebras with Weyl Groups of exceptional type, Preprint arXiv:0804.2602

Mathematics Department of Hunan University, Changsha China, 410082, E-Mail: z9491@yahoo.COM.CN 